\title{
Surface term effects on mass estimators
}

\author{
M. Membrado ${ }^{1}$ and A. F. Pacheco ${ }^{2,3}$ \\ 1 Departamento de Física Teórica, Universidad de Zaragoza, 50009 Zaragoza, Spain \\ e-mail: membrado@unizar.es \\ 2 Departamento de Física Teórica, Universidad de Zaragoza, 50009 Zaragoza, Spain \\ 3 BIFI, Instituto de Biofísica y Física de Sistemas Complejos, Universidad de Zaragoza, 50009 Zaragoza, Spain \\ e-mail: amalio@unizar.es
}

Received 19 November 2015 / Accepted 26 February 2016

\begin{abstract}
Context. We propose a way of estimating the mass contained in the volume occupied by a sample of galaxies in a virialized system. Aims. We analyze the influence of surface effects and the contribution of the cosmological constant terms on our mass estimations of galaxy systems.

Methods. We propose two equations that contain surface terms to estimate galaxy sample masses. When the surface terms are neglected, these equations provide the so-called virial and projected masses. Both equations lead to a single equation that allows sample masses to be estimated without the need for calculating surface terms. Sample masses for some nearest galaxy groups are estimated and compared with virialized masses determined from turn-around radii and results of a spherical infall model.

Results. Surface effects have a considerable effect on the mass estimations of the studied galaxy groups. According to our results, they lead sample masses of some groups to being less than half the virial mass estimations and even less than $10 \%$ of projected mass estimations. However, the contributions of cosmological constant terms to mass estimations are smaller than $2 \%$ for the majority of the virialized groups studied. Our estimations are in agreement with virialized masses calculated from turn-around radii. Virialized masses for complexes were found to be: $(8.9 \pm 2.8) \times 10^{11} M_{\odot}$ for the Milky Way - M 31; $(12.5 \pm 2.5) \times 10^{11} M_{\odot}$ for M $81-$ NGC 2403 ; $(21.5 \pm 7.7) \times 10^{11} M_{\odot}$. for Cantaurs A - M 83; and $(7.9 \pm 2.6) \times 10^{11} M_{\odot}$. for IC $324-$ Maffei.

Conclusions. The nearest galaxy groups located inside a sphere of $5 \mathrm{Mpc}$ have been addressed to explore the performance of our mass estimator. We have seen that surface effects make mass estimations of galaxy groups rather smaller than both virial and projected masses. In mass calculations, cosmological constant terms can be neglected; nevertheless, the collapse of cold dark matter leading to virialized structures is strongly affected by the cosmological constant. We have also seen that, if mass density were proportional to luminosity density on different scales in the Universe, the $5 \mathrm{Mpc}$ sphere would have a mean density close to that of the sphere region containing galaxies and systems of galaxies; thus, the rest of the sphere could contain regions of low-mass dark halos with similar mass density. This mass density would be about 4.5 times greater than that of the matter background of the Universe at present.
\end{abstract}

Key words. dark matter - dark energy

\section{Introduction}

For years, mass estimation of groups and clusters of galaxies has been one of the classical tasks of astronomers (see, for example: Zwicky 1933; Rood et al. 1970; Gott \& Turner 1977; Rood \& Dickel 1978; Rood 1981; Huchra \& Geller 1982; Geller 1984; Karachentsev 2005). Galactic kinematics, observations of $\mathrm{X}$-rays from hot intra-cluster gas or gravitational lensing are some of the techniques used to infer the mass of these systems.

From the redshifts and positions of galaxies, the virial mass is the classical mass estimator. Bahcall \& Tremaine (1981) studied the reliability of the masses derived from the virial estimator and proposed another estimator based on the projected mass. Heisler et al. (1985) extended the work of Bahcall \& Tremaine by exploring alternative mass estimators to the virial theorem for self-gravitating systems.

The virial theorem was introduced into astronomy by Zwicky (1933) in an approximate form to estimate the mass of the Coma cluster. It was applied to open star clusters by Chandrasekhar (1942), and to globular clusters by Schwarzschild (1954).

The virial theorem is derived by multiplying the steady Jeans equations by coordinate vectors and integrating them over a volume. If magnitudes are not null at the outer surface of this volume, a surface term appears. Mass estimation derived from this equation, when the surface term is neglected, is commonly known as the virial mass. This is because the virial theorem is usually applied without considering surface effects.

In the continuous model, when spherical symmetry and steady and static conditions are assumed, the virial theorem corresponds to the volume integral of the radial Jeans equation multiplied by the radial distance, $r$. The volume integral of the radial Jeans equation multiplied by $r^{2}$ has also been used by some authors (see, for example, Heisler et al. 1985). In fact, the mass estimation, which is derived when surface term is neglected, is known as the projected mass.

Equations equivalent to both volume integrals can be proposed to deal with a sample of $N$ galaxies. From these equations, the virial mass and the projected mass are also derived when their surface terms are not taken into account. If a system extends beyond the sample field, both masses could differ appreciably; in which case, the surface term contributions would be important.

In this work, we explore how the use of both equations allows the mass that is contained in the volume occupied by the sample to be estimated without calculating surface terms. Cosmological constant terms are also included in the equations. For 
isolated systems, surface terms are positive or null; hence, the sample mass must be smaller than, or equal to, both virial and projected masses; i.e., if surface terms are neglected, the mass is overestimated.

To estimate the mass of a sample, distances and radial velocities are required with sufficient precision for all its component galaxies (H I rotational velocity must also be known to derive indicative masses of galaxies). The Catalog of Neighboring Galaxies (Karachentsev et al. 2004) contains quality observational data within $10 \mathrm{Mpc}$. In fact, these data were used by Karachentsev (2005) to estimate virial and projected masses for galaxy groups within $5 \mathrm{Mpc}$. Our study will be applied to these groups.

In Sect. 2, the two equations that come from the volume integrals of the radial Jeans equation multiplied by $r$ (virial theorem) and by $r^{2}$ are presented in a continuous model for an isolated bound spherical system of galaxies. They are evaluated at a volume contained inside the whole system and thus, surface terms appear. The equation derived from them, which allows the mass inside the volume to be estimated without requiring surface term calculations, is also shown. Equations are also expressed as a function of projected quantities.

In Sect. 3, two other equations, equivalent to those of the continuous model, are proposed for dealing with a sample of $N$ galaxies. In these equations, cosmological constant terms are also included. Sections 3.1 and 3.2 are devoted to virial mass and projected mass estimators, respectively, derived from neglecting surface terms in these equations. Section 3.3 presents a third equation, which allows the mass inside the volume of the sample to be estimated without having to calculate surface terms.

Section 4 is devoted to applying the previously mentioned equations to neighbouring groups of galaxies to estimate virial, projected, and sample masses.

Section 5 deals with galaxy complexes composed of the galaxy groups addressed in this work. In Sect. 5.1, virialized masses of these complexes are calculated from measured turnaround radii. In Sect. 5.2, these virialized masses are compared with the sum of sample masses of the groups estimated in Sect. 4.

Finally, our conclusions are presented in Sect. 6.

\section{Mass estimators for isolated bound spherical systems of galaxies: continuous model}

In the continuous model, a system of galaxies is described by a phase space density $f_{\mathrm{g}}(\boldsymbol{r}, \boldsymbol{v}, t)$, where $\boldsymbol{r}, \boldsymbol{v}$, and $t$ are the spatial coordinate, velocity, and time. The mass density is then given by $\rho_{\mathrm{g}}(\boldsymbol{r}, t)=\int f_{\mathrm{g}}(\boldsymbol{r}, \boldsymbol{v}, t) \mathrm{d} \boldsymbol{v}$; so, the average in velocities of any quantity, $\eta(\boldsymbol{r}, \boldsymbol{v}, t)$, is calculated by $\bar{\eta}(\boldsymbol{r}, t)=$ $\left[\int \eta(\boldsymbol{r}, \boldsymbol{v}, t) f_{\mathrm{g}}(\boldsymbol{r}, \boldsymbol{v}, t) \mathrm{d} \boldsymbol{v}\right] / \rho_{\mathrm{g}}(\boldsymbol{r})$. Let us assume that the evolution of the phase space density is described by the collisionless Boltzmann equation. The integration over all velocities of the product of the Boltzmann equation with the velocity vector $v$ leads to the Jeans equations.

The radial Jeans equation, assuming spherical symmetry and steady and static conditions $(\overline{\boldsymbol{v}}=0)$, is given by (see, for example, Binney \& Tremaine 1987, p. 198)

$\frac{\mathrm{d}}{\mathrm{d} r}\left[\rho_{\mathrm{g}} \overline{v_{r}^{2}}\right]+\frac{\rho_{\mathrm{g}}}{r}\left[2 \overline{v_{r}^{2}}-\left(\overline{v_{\theta}^{2}}+\overline{v_{\varphi}^{2}}\right]=-\rho_{\mathrm{g}} \frac{\mathrm{d} \phi}{\mathrm{d} r}\right.$,

where $\phi$ is the gravitational potential generated by matter and dark energy. In Eq. (1), subindexes $r, \theta$, and $\varphi$ indicate radial, polar, and azimuthal components. As $\overline{v_{r}}=\overline{v_{\theta}}=\overline{v_{\varphi}}=0$, velocity dispersions of galaxies fulfill $\sigma_{r}^{2}=\overline{v_{r}^{2}}, \sigma_{\theta}^{2}=\overline{v_{\theta}^{2}}$ and $\sigma_{\varphi}^{2}=\overline{v_{\varphi}^{2}}$; and hence, $\sigma^{2}=\overline{v_{r}^{2}}+\overline{v_{\theta}^{2}}+\overline{v_{\varphi}^{2}}$. In the following, we use $\sigma_{r}^{2}, \sigma_{\theta}^{2}$, and $\sigma_{\varphi}^{2}$ instead of $\overline{v_{r}^{2}}, \overline{v_{\theta}^{2}}$ and $\overline{v_{\varphi}^{2}}$.

If dark energy is treated from a hydrodynamical point of view and the cosmological constant model is assumed, $\phi$ can be derived from

$\nabla^{2} \phi=4 \pi G\left[\rho+\frac{1}{c^{2}}\left(\rho_{\Lambda}+3 p_{\Lambda}\right)\right]$.

In Eq. (2), $\rho$ is the mass density of matter and $\rho_{\Lambda}$ and $p_{\Lambda}$, the energy density and pressure of the dark energy $\left(\rho_{\Lambda}=\frac{c^{4}}{4 \pi G} \Lambda=-p_{\Lambda}\right.$, $\Lambda$ being the cosmological constant). Thus, taking into account the spherical symmetry of the system,

$\frac{\mathrm{d} \phi}{\mathrm{d} r}=\frac{G m}{r^{2}}-\frac{8 \pi G \rho_{\Lambda}}{3 c^{2}} r$,

where $m(r)$ is the mass of matter enclosed up to $r$, i.e.

$m(r)=4 \pi \int_{0}^{r} \rho r^{\prime 2} \mathrm{~d} r^{\prime}$.

\subsection{The virial theorem}

The virial theorem is derived by multiplying Eq. (1) by $4 \pi r^{3}$ and integrating from $r=0$ up to some $r=R$. Thus,

$$
\begin{aligned}
4 \pi R^{3} \rho_{\mathrm{g}}(R) \sigma_{r}^{2}(R)= & M_{\mathrm{g}}\left\langle\sigma^{2}\right\rangle_{M_{\mathrm{g}}} \\
& -G M_{\mathrm{g}}\left\langle\frac{m}{r}\right\rangle_{M_{\mathrm{g}}}+\frac{8 \pi G \rho_{\Lambda}}{3 c^{2}} M_{\mathrm{g}}\left\langle r^{2}\right\rangle_{M_{\mathrm{g}}} .
\end{aligned}
$$

In Eq. (5), $M_{\mathrm{g}}$ is the mass of galaxies contained in the sphere of radius $R$; and for any quantity $\eta(r)$,

$\langle\eta\rangle_{M_{\mathrm{g}}}=\frac{1}{M_{\mathrm{g}}} \int_{0}^{R} \int_{0}^{2 \pi} \int_{0}^{\pi} \rho_{\mathrm{g}} \eta r^{2} \sin \theta \mathrm{d} \theta \mathrm{d} \varphi \mathrm{d} r$.

When we deal with a galaxy system, its mass is defined by the spatial extension of the sample. Hence, if the system extends beyond the observed field and the surface term in Eq. (5) is neglected, the mass obtained up to its observed extension is overestimated (the pressure at the outer surface of the observed field causes the mass needed to keep the system in equilibrium to be smaller).

One way of dealing with the surface term of Eq. (5) was developed by The \& White (1986). For the Coma cluster, they estimated the maximum possible value of the surface term by determining the largest value of $\sigma_{r}^{2}$ at $R=5.4 h_{50}^{-1} \mathrm{Mpc}\left(h_{50}=\right.$ $H_{0} / 50 \mathrm{~km} \mathrm{~s}^{-1} \mathrm{Mpc}^{-1} ; H_{0}$ being the Hubble function at present), consistent with the observational data. They concluded that the surface term should be smaller than $42 \%$ of the kinetic term, and assumed a value of 0.21 times the kinetic term. Rines et al. (2003) also calculated the overestimation of the virial mass when the system does not lie entirely within the virial radius for the clusters treated in the CAIRNS (Cluster and Infall Region Nearby Survey) project.

An estimation of an upper limit for the surface term can also be derived assuming a singular isothermal sphere. The fact that bound gravitational systems show decreasing velocity dispersions with radius means that isothermal spheres have a greater surface term. If galaxies are assumed to trace the mass, the mass density of galaxies, $\rho_{\mathrm{g}}$, and matter, $\rho$, would be given by

$\rho_{\mathrm{g}}=\frac{M_{\mathrm{g}}}{4 \pi R r^{2}}$,
$\rho=\frac{M}{4 \pi R r^{2}}$, 
$M_{\mathrm{g}}$ and $M$ being the mass of galaxies and the mass of matter enclosed up to $R$. When dark energy is not taken into account, Eqs. (7)-(8) are solutions of the Jeans Eq. (1) if

$M=\frac{\sigma^{2}-\sigma_{r}^{2}}{G} R$.

This result is also derived from Eq. (5). However, when the surface term in (5) is neglected, the mass derived does not correspond to that shown in Eq. (9); in fact, it is overestimated by a factor $\sigma^{2} /\left(\sigma^{2}-\sigma_{r}^{2}\right)$. Hence, the surface term contributes appreciably to the mass. For isotropic orbits, Eq. (9) tells us that $M=\left(2 \sigma_{r}^{2} / G\right) R$. In that case, $\sigma_{r}^{2} / \sigma^{2}=1 / 3$, which can be compared with the assumption of The \& White (1986) for the surface term.

When cosmological constant effects are taken into account, $\rho_{\mathrm{g}}$ and $\rho$ given by Eqs. (7) and (8) do not fulfill the Jeans Eq. (1). In any case, assuming these mass densities, an estimation for the mass inside a sphere of radius $R$ can be derived from Eq. (5). This equation leads to

$M=\frac{\sigma^{2}-\sigma_{r}^{2}}{G} R+\frac{8 \pi \rho_{\Lambda}}{9 c^{2}} R^{3}$.

Equation (10) was used by Membrado \& Aguerri (2004) and Membrado \& Pacheco (2014) in the study of the collapse of spherical mass shells in a cosmological background of matter and cosmological constant. This equation allowed the virial radius of a shell to be estimated from its turn-around radius.

\subsection{A way of avoiding surface terms}

When the Jeans Eq. (1) is multiplied by $r^{4}$ and integrated from $r=0$ up to $r=R$, the equation obtained is

$R^{4} \rho_{\mathrm{g}}(R) \sigma_{r}^{2}(R)=\int_{0}^{R} r^{3} \rho_{\mathrm{g}}\left[\sigma^{2}+\sigma_{r}^{2}\right] \mathrm{d} r-\int_{0}^{R} r^{4} \rho_{\mathrm{g}} \frac{\mathrm{d} \phi}{\mathrm{d} r} \mathrm{~d} r$.

Then, using Eqs. (3) and (6) in Eq. (11), we obtain

$$
\begin{aligned}
4 \pi R^{4} \rho_{\mathrm{g}}(R) \sigma_{r}^{2}(R)= & M_{\mathrm{g}}\left\langle r\left[\sigma^{2}+\sigma_{r}^{2}\right]\right\rangle_{M_{\mathrm{g}}} \\
& -G M_{\mathrm{g}}\langle m\rangle_{M_{\mathrm{g}}}+\frac{8 \pi G \rho_{\Lambda}}{3 c^{2}} M_{\mathrm{g}}\left\langle r^{3}\right\rangle_{M_{\mathrm{g}}} .
\end{aligned}
$$

In the case of assuming singular isothermal spheres (see Eqs. (7) and (8)), Eq. (9) is also derived from (12) when dark energy is not taken into account. If the surface term is neglected, the mass is overestimated by a factor $\left(\sigma^{2}+\sigma_{r}^{2}\right) /\left(\sigma^{2}-\sigma_{r}^{2}\right)$. We should also point out that the contribution of the cosmological constant term is $3 / 2$ times greater than that shown in Eq. (10). This is because Eqs. (7) and (8) do not fulfill the Jeans Eq. (1).

The bonus of Eq. (12) is that its surface term can be eliminated by using the virial theorem given by Eq. (5). Thus,

$$
\begin{aligned}
& R\left[\left\langle\sigma^{2}\right\rangle_{M_{\mathrm{g}}}-G\left\langle\frac{m}{r}\right\rangle_{M_{\mathrm{g}}}+\frac{8 \pi G \rho_{\Lambda}}{3 c^{2}}\left\langle r^{2}\right\rangle_{M_{\mathrm{g}}}\right]= \\
& \left\langle r\left[\sigma^{2}+\sigma_{r}^{2}\right]\right\rangle_{M_{\mathrm{g}}}-G\langle m\rangle_{M_{\mathrm{g}}}+\frac{8 \pi G \rho_{\Lambda}}{3 c^{2}}\left\langle r^{3}\right\rangle_{M_{\mathrm{g}}}
\end{aligned}
$$

Following with the example of singular isothermal spheres, the mass given by Eq. (9) is again derived from (13) when dark energy terms are not considered. When those terms are not neglected, they lead to a contribution which is half that shown in Eq. (10).

\subsection{Using projected quantities}

From observations of galaxies, we have access to their velocities in the line of sight, to their projected separations and, in some cases, to their distances. Therefore, it is useful to express Eqs. (5) and (12) in a manner in which we can include observational measurements. This can be done by using projected quantities.

Let us consider a point that has a position vector $\boldsymbol{r}$ with respect to the mass centre, and a position vector $\boldsymbol{R}$ with respect to the observer. Taking the line of sight of the mass centre as the $z-$ direction, we can define the polar angle with respect to the mass centre, $\theta$, and the polar angle with respect to the observer, $\Theta$; these angles fulfill $\cos \theta=\hat{r} \cdot \hat{z}$ and $\cos \Theta=\hat{R} \cdot \hat{z}$. At $\boldsymbol{r}$, we have a distribution of velocities given by the distribution function $f(\boldsymbol{r}, \boldsymbol{v})$.

At the point $\boldsymbol{r}$, the component of the velocity in the line of sight of the mass centre, $v_{\mathrm{z}}$, as a function of the radial, polar, and azimuthal components of the velocity is

$v_{\mathrm{z}}=-v_{\theta} \sin \theta+v_{r} \cos \theta$.

Hence, as $\sigma_{\mathrm{z}}^{2}=\overline{v_{\mathrm{z}}^{2}}-\left(\overline{v_{\mathrm{z}}}\right)^{2}$ and $\overline{v_{\mathrm{z}}}=0$,

$\left\langle\sigma_{\mathrm{z}}^{2}\right\rangle_{M_{\mathrm{g}}}=\frac{1}{3}\left\langle\sigma_{r}^{2}\right\rangle_{M_{\mathrm{g}}}+\frac{2}{3}\left\langle\sigma_{\theta}^{2}\right\rangle_{M_{\mathrm{g}}}$.

For the sake of simplicity, we could assume

$\sigma_{\theta}=\sigma_{\varphi}$

and a constant anisotropy parameter $\beta$, given by

$\beta=1-\frac{\sigma_{\theta}^{2}(r)}{\sigma_{r}^{2}(r)}$.

Hence,

$\left\langle\sigma_{\mathrm{z}}^{2}\right\rangle_{M_{\mathrm{g}}}=\frac{1}{3}\left\langle\sigma^{2}\right\rangle_{M_{\mathrm{g}}}=\left[1-\frac{2 \beta}{3}\right]\left\langle\sigma_{r}^{2}\right\rangle_{M_{\mathrm{g}}}$.

With respect to the component of the velocity in the line of sight, $v_{\text {los }}$, it is easy to realize that $v_{\text {los }}=-v_{\theta} \sin (\theta-\Theta)+v_{r} \cos (\theta-\Theta)$. When isotropy is assumed (i.e. $\beta=0$ ) and as $\overline{v_{\text {los }}}=0, \sigma_{\text {los }}^{2}=$ $\overline{v_{\mathrm{los}}^{2}}=\sigma_{r}^{2}=\sigma_{\theta}^{2}=\sigma_{\varphi}^{2}=\sigma_{\mathrm{z}}^{2}$. Thus,

$\left\langle\sigma_{\text {los }}^{2}\right\rangle_{M_{\mathrm{g}}}=\left\langle\sigma_{\mathrm{z}}^{2}\right\rangle_{M_{\mathrm{g}}}=\frac{1}{3}\left\langle\sigma^{2}\right\rangle_{M_{\mathrm{g}}}$.

Now, let us have a look at the second term on the right hand side of Eq. (5). Taking into account that

$r_{\perp}=r \sin \theta$

then,

$\left\langle\frac{m}{r_{\perp}}\right\rangle_{M_{\mathrm{g}}}=\frac{\pi}{2}\left\langle\frac{m}{r}\right\rangle_{M_{\mathrm{g}}}$.

With respect to the cosmological constant term of Eq. (5), the use of Eq. (20) leads to

$\left\langle r_{\perp}^{2}\right\rangle_{M_{\mathrm{g}}}=\frac{2}{3}\left\langle r^{2}\right\rangle_{M_{\mathrm{g}}}$

Hence, using Eqs. (18), (21), and (22) in Eq. (5), the virial theorem for a steady and static gravitational system, assuming spherical symmetry and a constant anisotropy parameter, reads as

$$
\begin{aligned}
4 \pi R^{3} \rho_{\mathrm{g}}(R) \sigma_{r}^{2}(R)= & 3 M_{\mathrm{g}}\left\langle\sigma_{\mathrm{z}}^{2}\right\rangle_{M_{\mathrm{g}}} \\
& -\frac{2}{\pi} G M_{\mathrm{g}}\left\langle\frac{m}{r_{\perp}}\right\rangle_{M_{\mathrm{g}}}+\frac{4 \pi G \rho_{\Lambda}}{c^{2}} M_{\mathrm{g}}\left\langle r_{\perp}^{2}\right\rangle_{M_{\mathrm{g}}} .
\end{aligned}
$$

If isotropy is assumed, Eq. (19) can be used instead of (18). 
With respect to Eq. (12), the first and third terms on the right hand side of the equation can also be expressed as functions of projected quantities. From Eqs. (14) and (20),

$\left\langle r_{\perp} \sigma_{\mathrm{z}}^{2}\right\rangle_{M_{\mathrm{g}}}=\frac{\pi}{16}\left\langle r \sigma_{r}^{2}\right\rangle_{M_{\mathrm{g}}}+\frac{3 \pi}{16}\left\langle r \sigma_{\theta}^{2}\right\rangle_{M_{\mathrm{g}}}$.

Thus, if a constant anisotropy parameter given by Eqs. (16) and (17) is assumed,

$\left\langle r_{\perp} \sigma_{\mathrm{z}}^{2}\right\rangle_{M_{\mathrm{g}}}=\frac{\pi}{32} \frac{(4-3 \beta)}{(2-\beta)}\left\langle r\left[\sigma^{2}+\sigma_{r}^{2}\right]\right\rangle_{M_{\mathrm{g}}} ;$

and in the case of isotropy $\left(\beta=0\right.$ and $\left.\sigma_{\mathrm{z}}^{2}=\sigma_{\mathrm{los}}^{2}\right)$,

$\left\langle r_{\perp} \sigma_{\mathrm{los}}^{2}\right\rangle_{M_{\mathrm{g}}}=\frac{\pi}{16}\left\langle r\left[\sigma^{2}+\sigma_{r}^{2}\right]\right\rangle_{M_{\mathrm{g}}}$.

With respect to the third term of Eq. (12), from Eq. (20),

$\left\langle r_{\perp}^{3}\right\rangle_{M_{\mathrm{g}}}=\frac{3 \pi}{16}\left\langle r^{3}\right\rangle_{M_{\mathrm{g}}}$

Now, using Eqs. (25) and (27), Eq. (12) reads as

$$
\begin{aligned}
4 \pi R^{4} \rho_{\mathrm{g}}(R) \sigma_{r}^{2}(R)= & \frac{32}{\pi} \frac{(2-\beta)}{(4-3 \beta)} M_{\mathrm{g}}\left\langle r_{\perp} \sigma_{\mathrm{z}}^{2}\right\rangle_{M_{\mathrm{g}}} \\
& -G M_{\mathrm{g}}\langle m\rangle_{M_{\mathrm{g}}}+\frac{128 G \rho_{\Lambda}}{9 c^{2}} M_{\mathrm{g}}\left\langle r_{\perp}^{3}\right\rangle_{M_{\mathrm{g}}}
\end{aligned}
$$

\section{Mass estimators for isolated bound spherical systems of galaxies: discrete model}

Let us suppose now that an isolated galaxy system extends beyond the field occupied by a sample of $N$ galaxies. The masses, positions, and velocities of galaxies are denoted by $\left[m_{\mathrm{g}}\right]_{i}, \boldsymbol{R}_{i}$, and $\boldsymbol{V}_{i}$. The total mass of the $N$ galaxies is $M_{\mathrm{g}}=\sum_{i=1}^{N}\left[m_{\mathrm{g}}\right]_{i}$; and the mass of matter inside the extension of the sample, $M$. In the intergalactic medium, the distribution of dark matter is unknown; therefore, the mass distribution in galaxy systems is also unknown. In this work, for the sake of simplicity, we assume that galaxies trace the mass.

For the sample of galaxies, the position and velocity of the centre of mass are

$\left\langle\boldsymbol{R}_{i}\right\rangle_{\mathrm{M}}=\frac{1}{M_{\mathrm{g}}} \sum_{i=1}^{N}\left[m_{\mathrm{g}}\right]_{i} \boldsymbol{R}_{i}, \quad\left\langle\boldsymbol{V}_{i}\right\rangle_{\mathrm{M}}=\frac{1}{M_{\mathrm{g}}} \sum_{i=1}^{N}\left[m_{\mathrm{g}}\right]_{i} \boldsymbol{V}_{i}$.

Hence, the positions and velocities of galaxies relative to the mass centre are

$\boldsymbol{r}_{i}=\boldsymbol{R}_{i}-\left\langle\boldsymbol{R}_{i}\right\rangle_{\mathrm{M}}, \quad \boldsymbol{v}_{i}=\boldsymbol{V}_{i}-\left\langle\boldsymbol{V}_{i}\right\rangle_{\mathrm{M}}$.

The component of $\boldsymbol{r}_{i}$ perpendicular to the line of sight of the mass center, $\hat{z} \equiv\left\langle\boldsymbol{R}_{i}\right\rangle_{\mathrm{M}} /\left|\left\langle\boldsymbol{R}_{i}\right\rangle_{\mathrm{M}}\right|$, is denoted by $\left[\boldsymbol{r}_{i}\right]_{\perp}$; and the projection of $\boldsymbol{v}_{i}$ on the line of sight of the mass centre, by $\left[\boldsymbol{v}_{i}\right]_{\mathrm{z}}$.

\subsection{Virial mass estimator}

We will assume that the system shows spherical symmetry and a constant anisotropy parameter. In this case, an expression for the virial theorem for the sample of the $N$ galaxies, similar to that given for a continuous model by Eq. (23), is:

$$
\begin{aligned}
\left\langle S_{\mathrm{vir}}\left(R_{\mathrm{S}}\right)\right\rangle_{t} & =3\left\langle\sum_{i=1}^{N}\left[m_{\mathrm{g}}\right]_{i}\left[v_{i}\right]_{\mathrm{Z}}^{2}\right\rangle_{t} \\
- & \frac{2}{\pi} G\left\langle\sum_{i=1}^{N} \frac{\left[m_{\mathrm{g}}\right]_{i}[\mathcal{M}]_{i}}{\left[r_{i}\right]_{\perp}}\right\rangle_{t}+\frac{4 \pi G \rho_{\Lambda}}{c^{2}}\left\langle\sum_{i=1}^{N}\left[m_{\mathrm{g}}\right]_{i}\left[r_{i}\right]_{\perp}^{2}\right\rangle .
\end{aligned}
$$

In Eq. (31), $R_{\mathrm{S}}$ is the radius of the sphere containing the $N$ galaxies. $S_{\text {vir }}\left(R_{\mathrm{S}}\right)$ is a surface term (see surface term of Eq. (23)) evaluated at a distance $R_{\mathrm{s}}$. [M] $]_{i}$ is the mass covered by a spherical surface of radius $r_{i}$. Finally, the brackets stand for time averages, which are taken to obtain steady magnitudes. It is, of course, not possible to obtain time averages from observations. However, the sums of individual contributions at present on the right hand side of Eq. (31) would introduce a kind of averaging. It is expected that the magnitudes at the present epoch fulfill the virial theorem (31) reasonably well.

The second term on the right hand side of Eq. (31) comes from the trace of the Chandrasekhar gravitational potential energy tensor in Eq. (5) (see, for example, Binney \& Tremaine 1987 , p. 67). However, it should be noted that the gravitational energy is more commonly used. Proof of the virial theorem in the discrete model using gravitational energy and projected magnitudes can be seen, for example, in Heisler et al. (1985). Here, the surface term is not taken into account and the cosmological constant effects are neglected. When those contributions are considered, the virial theorem can be expressed as

$$
\begin{aligned}
\left\langle S_{\mathrm{vir}}\left(R_{\mathrm{s}}\right)\right\rangle_{t}= & 3\left\langle\sum_{i=1}^{N}\left[m_{\mathrm{g}}\right]_{i}\left[v_{i}\right]_{\mathrm{z}}^{2}\right\rangle_{t} \\
& -\frac{2}{\pi} \frac{G M}{M_{\mathrm{g}}}\left\langle\sum_{i=1}^{N} \sum_{j<i} \frac{\left[m_{\mathrm{g}}\right]_{i}\left[m_{\mathrm{g}}\right]_{j}}{\left[r_{i j}\right]_{\perp}}\right\rangle_{t} \\
& +\frac{4 \pi G \rho_{\Lambda}}{c^{2}} \sum_{i=1}^{N}\left\langle\left[m_{\mathrm{g}}\right]_{i}\left[r_{i}\right]_{\perp}^{2}\right\rangle_{t},
\end{aligned}
$$

where

$r_{i j}=\left|\boldsymbol{r}_{i j}\right|=\left|\boldsymbol{r}_{i}-\boldsymbol{r}_{i}\right|=\left|\boldsymbol{R}_{i}-\boldsymbol{R}_{j}\right|$.

Some authors use $\left[r_{i j}\right]_{\perp}$ as the component of $\boldsymbol{r}_{i j}$ perpendicular to the line of sight of the ith galaxy; others, as the component of $\boldsymbol{r}_{i j}$ perpendicular to the line of sight of the mass center.

Equations (31) or (32) tells us that galaxy contributions should be weighted by galaxy masses. In this respect, indicative masses can be derived from HI rotation velocities. Some authors take the same mass for all galaxies. This would introduce another kind of averaging in the equations. In this sense, the $N$-body simulations developed by Heisler et al. (1985) indicated that their estimators worked well, even if galaxies had a range of masses.

Neglecting the surface term and the contribution of the cosmological constant term, mass estimators derived from different expressions for the virial theorem have been extensively used (see, for example: Limber \& Mathews 1960; Huchra \& Geller 1982; Carlberg et al. 1996; Karachentsev 2005). Chernin et al. (2012) included the cosmological constant term in mass estimators. They concluded that the cosmological constant term must be taken into account in groups of galaxies, but its contribution is negligible in galaxy clusters.

The aim of this work is to estimate the effects of surface terms on mass estimators when applied to a galaxy system. To develop this, accurate distances and radial velocities are required for all galaxies in the system. Moreover, to estimate the mass of each galaxy, the $\mathrm{H}$ I rotational velocity must be known. All these data are available for galaxy systems within $10 \mathrm{Mpc}$ from the Catalog of Neighboring Galaxies (Karachentsev et al. 2004). In fact, the quantity and quality of these observational data allowed Karachentsev (2005) to determine masses for galaxy groups 
within 5 Mpc (Milky Way, M31, M81, NGC 2403, Centaurus A, M 83, IC 342, Maffei, Sculptor Filament and Canes Venatici I Cloud). In this paper, we also deal with these neighbouring galaxy systems.

Each one of these groups has a massive galaxy, which contains nearly all the mass of galaxies of the system. In this work, we assume that the mass centre of a group coincides with the centre of its most massive galaxy, denoted by $i=1$ galaxy.

For a sample of $N$ galaxies with a main galaxy that dominates, the virial theorem given by Eq. (31) is applied to the rest of the $N-1$ galaxies (note that $r_{1}=0$ and $[\mathcal{M}]_{1}=0$ ). To estimate (31), we assume that such $N-1$ galaxies have the same mass; i.e.

$\left[m_{\mathrm{g}}\right]_{i=1} \gg\left[m_{\mathrm{g}}\right]_{i>1}$,

$\left[m_{\mathrm{g}}\right]_{i>1}=M_{\mathrm{g}}^{c} /(N-1)$,

$M_{\mathrm{g}}=\left[m_{\mathrm{g}}\right]_{1}+M_{\mathrm{g}}^{c}$.

Thus, the virial theorem reads as

$S_{\mathrm{vir}}\left(R_{\mathrm{s}}\right)=3 M_{\mathrm{g}}^{c}\left[\sigma_{\mathrm{z}}^{2}\right]-\frac{G M M_{\mathrm{g}}^{c}}{\left[R_{\mathrm{H}}\right]}+\frac{4 \pi G \rho_{\Lambda}}{c^{2}} M_{\mathrm{g}}^{c}\left[R_{\perp}^{2}\right]$.

The kinetic term of Eq. (31) has been approximated by the kinetic term of Eq. (37); in this term, we take $\left[\sigma_{\mathrm{z}}^{2}\right] \approx\left[\sigma_{\mathrm{los}}^{2}\right]$ (both quantities that coincide in the case of isotropy; see Eq. (19)), where $\left[\sigma_{\mathrm{los}}^{2}\right]^{1 / 2}$ is the dispersion of line of sight velocities with respect to that of the sample centroid. In Eq. (37), $\left[R_{\mathrm{H}}\right]$ is the mean harmonic radius, and $\left[R_{\perp}^{2}\right]$, the mean square projected radius. Thus,

$\left[\sigma_{\mathrm{z}}^{2}\right] \approx\left[\sigma_{\mathrm{los}}^{2}\right]=\frac{1}{N} \sum_{i=1}^{N}\left[\left[V_{i}\right]_{\mathrm{los}}-\frac{1}{N} \sum_{j=1}^{N}\left[V_{j}\right]_{\mathrm{los}}\right]^{2}$,

$\left[R_{\mathrm{H}}\right]=\frac{\pi}{2}\left[\frac{1}{N-1} \sum_{i=2}^{N} \frac{1}{\left[r_{i}\right]_{\perp}}\left(\frac{[\mathcal{M}]_{i}}{M}\right)\right]^{-1}$,

$\left[R_{\perp}^{2}\right]=\frac{1}{N-1} \sum_{i=2}^{N}\left[r_{i}\right]_{\perp}^{2}$

To deal with $[\mathcal{M}]_{i}$ in Eq. (39), we explore three models: model PM takes a central point mass; model MG considers that the companions to the main galaxy have the same mass (see Eq. (35)); and, model EM assumes equal mass for all galaxies. These models are used for reasons of simplicity and because they introduce another type of averaging in the calculations.

In Sect. 4, neighbouring galaxy groups are dealt with. The results in that section seem to indicate that $\left[R_{\mathrm{H}}\right]$ shows a dependence on galaxy positions, which is stronger than that of the mass contribution of the companions to the main galaxy. Thus, some groups with a massive main galaxy are better represented by a harmonic radius that is calculated assuming equal mass galaxies. In contrast, other groups with a main galaxy, but where companions contribute appreciably to the mass, are better represented by a harmonic radius calculated assuming central point mass.

All models $[\mathcal{M}]_{i}$ fulfill

$[\mathcal{M}]_{i=1}=0$.

When galaxies are numbered according to their distances from the main galaxy, $[\mathcal{M}]_{i>1}$ 's from models PM, MG, and EM are given by

$[\mathcal{M}]_{i>1} \equiv\left[\mathcal{M}_{\mathrm{PM}}\right]_{i>1}=M$,

$[\mathcal{M}]_{i>1} \equiv\left[\mathcal{M}_{\mathrm{MG}}\right]_{i>1}=\frac{M}{M_{\mathrm{g}}}\left[\left[m_{\mathrm{g}}\right]_{1}+(i-2) \frac{M_{\mathrm{g}}^{c}}{(N-1)}\right]$,

$[\mathcal{M}]_{i>1} \equiv\left[\mathcal{M}_{\mathrm{EM}}\right]_{i>1}=\frac{M}{M_{\mathrm{g}}}\left[(i-1) \frac{M_{\mathrm{g}}}{N}\right]$.

It can be seen that when $M_{\mathrm{g}}^{c} \ll\left[m_{\mathrm{g}}\right]_{1}$ (main galaxy dominates the mass), Eq. (43) tends to Eq. (42); and, when $\left[m_{\mathrm{g}}\right]_{1}=M_{\mathrm{g}}^{c} /(N-$ 1) (all galaxies have the same mass), Eq. (43) coincides with Eq. (44).

Equations (43) and (44) are not directly used in Eq. (39). To introduce another kind of averaging in the discrete model, these equations are averaged in the $(N-1)$ companions to the main galaxy. Thus, for models MG and EM, we take

$$
\begin{aligned}
{[\mathcal{M}]_{i>1} } & \equiv\left[\mathcal{M}_{\mathrm{MG}}\right]=\frac{1}{N-1} \sum_{i=2}^{N}\left[\mathcal{M}_{\mathrm{MG}}\right]_{i} \\
& =M\left[\frac{\left[m_{\mathrm{g}}\right]_{1}}{M_{\mathrm{g}}}+\frac{1}{2} \frac{(N-2)}{(N-1)} \frac{M_{\mathrm{g}}^{c}}{M_{\mathrm{g}}}\right], \\
{[\mathcal{M}]_{i>1} } & \equiv\left[\mathcal{M}_{\mathrm{EM}}\right]=\frac{1}{N-1} \sum_{i=1}^{N}\left[\mathcal{M}_{\mathrm{EM}}\right]_{i}=\frac{1}{2} M .
\end{aligned}
$$

When using Eq. (42) in Eq. (39), the harmonic radius for model $\mathrm{PM}$ is given by

$\left[R_{\mathrm{H}}\right]_{\mathrm{PM}}=\frac{\pi}{2}\left[\frac{1}{N-1} \sum_{i=2}^{N} \frac{1}{\left[r_{i}\right]_{\perp}}\right]^{-1}$.

This is the case for galaxies orbiting around a point mass. The virial theorem for this system, neglecting the cosmological constant term and surface term, was addressed, for example, by Bahcall \& Tremaine (1981).

The harmonic radius for model MG can be derived using Eqs. (45) in (39). Thus

$\left[R_{\mathrm{H}}\right]_{\mathrm{MG}}=\left[\frac{\left[m_{\mathrm{g}}\right]_{1}}{M_{\mathrm{g}}}+\frac{1}{2} \frac{(N-2)}{(N-1)} \frac{M_{\mathrm{g}}^{c}}{M_{\mathrm{g}}}\right]^{-1}\left[R_{\mathrm{H}}\right]_{\mathrm{PM}}$.

The greater the difference between $\left[R_{\mathrm{H}}\right]_{\mathrm{MG}}$ and $\left[R_{\mathrm{H}}\right]_{\mathrm{PM}}$, the greater the mass contributions of the companions to the main galaxy.

And, when Eq. (46) is used in Eq. (39),

$\left[R_{\mathrm{H}}\right]_{\mathrm{EM}}=2\left[R_{\mathrm{H}}\right]_{\mathrm{PM}}$

(note that when all galaxies have the same mass, $\left[R_{\mathrm{H}}\right]_{\mathrm{EM}}=$ $\left[R_{\mathrm{H}}\right]_{\mathrm{MG}}$ ). Some authors (see, for example, Karachentsev 2005), instead of using $\left[R_{\mathrm{H}}\right]_{\mathrm{EM}}$, use

$\left[R_{\mathrm{H}}\right]_{\mathrm{K}}=\frac{2 N}{N-1}\left[R_{\mathrm{H}}\right]_{\mathrm{PM}}$.

Equation (50) can be deduced from Eq. (39), taking for $[\mathcal{M}]_{i}$, the average of $\left[\mathcal{M}_{\mathrm{EM}}\right]_{i}$ (see Eq. (44)) in the $N$ galaxies.

Hereafter, $[M]$ will be used to represent mass estimations which do not take into account cosmological constant terms. Estimations of mass obtained considering cosmological constant terms will be indicated by $\{M\}$. 
In the case of neglecting the surface term of Eq. (37), an estimator for the mass inside the sphere of radius $R_{\mathrm{s}}$ is

$\left\{M_{\mathrm{vir}}\right\}=\left[M_{\mathrm{vir}}\right]\left(1+\left[f_{\mathrm{vir}}\right]\right)$,

where

$$
\begin{aligned}
{\left[M_{\mathrm{vir}}\right] } & =\frac{3}{G}\left[\sigma_{\mathrm{los}}^{2}\right]\left[R_{\mathrm{H}}\right], \\
{\left[f_{\mathrm{vir}}\right] } & =\frac{4 \pi G \rho_{\Lambda}}{3 c^{2}} \frac{\left[R_{\perp}^{2}\right]}{\left[\sigma_{\mathrm{los}}^{2}\right]}=\frac{1}{2} H_{0}^{2} \Omega_{\Lambda} \frac{\left[R_{\perp}^{2}\right]}{\left[\sigma_{\mathrm{los}}^{2}\right]} .
\end{aligned}
$$

In Eq. (53), $H_{0}$ is the Hubble function at present $\left(H_{0}^{2}=\right.$ $\left[8 \pi G \rho_{0}\right] /\left[3 c^{2}\right] ; \rho_{0}$ being the energy density of the present Universe), and $\Omega_{\Lambda}$ is the fraction of dark energy in the Universe at present $\left(\Omega_{\Lambda}=\rho_{\Lambda} / \rho_{0}\right)$. Values for $\Omega_{\Lambda}$ and $H_{0}$ can be taken from Spergel et al. (2003); in this work we assume $H_{0}=$ $71 \mathrm{~km} \mathrm{~s}^{-1} \mathrm{Mpc}^{-1}$ and $\Omega_{\Lambda}=0.73$.

Equation (51) can be compared with the following virial mass estimator proposed by Chernin et al. (2012) which includes a cosmological constant term:

$\left\{M_{\mathrm{C}}\right\}=\frac{v^{2} r}{G}\left[1+\frac{8 \pi G \rho_{\Lambda}}{3 c^{2}}\left(\frac{r}{v}\right)^{2}\right]=\frac{v^{2} r}{G}\left(1+f_{\mathrm{C}}\right)$.

In Eq. (54), $r$ and $v$ are the characteristic size and velocity of the system. For groups of galaxies, these authors took $r \approx 1 \mathrm{Mpc}$ and $v \approx 70 \mathrm{~km} \mathrm{~s}^{-1}$, so $f_{\mathrm{C}} \approx 0.75$. For clusters of galaxies, they considered $r \approx 5 \mathrm{Mpc}$ and $v \approx 1000 \mathrm{~km} \mathrm{~s}^{-1}$ leading to $f_{\mathrm{C}} \approx$ 0.09. Hence, according to Chernin et al. (2012), the contribution of the cosmological constant term is negligible in the estimation of virial masses of galaxy clusters, but considerable in galaxy groups.

It is obvious that when observational measurements are used in mass estimators that do not include $\Lambda$-terms, the masses derived are not those in a $\Lambda=0$ Universe. Hence, the contribution of $\Lambda$-terms in mass estimators should not be confused with the effect of the cosmological constant. To estimate the actual effect of $\Lambda$, it would be necessary to know positions and velocities of galaxies in a $\Lambda=0$ Universe (such data cannot be obtained from observational measurements, and only simulations of structure formation or infall models in a $\Lambda=0$ Universe could provide that information).

We evaluated the surface term $S\left(R_{\mathrm{S}}\right)$ at the distance of the farthest galaxy of the sample from the centre of the massive dominant galaxy; i.e. at

$R_{\mathrm{S}}=\max \left\{\left|\boldsymbol{r}_{1}\right|, \ldots,\left|\boldsymbol{r}_{N}\right|\right]$.

Equation (55) has been used, for example, by The \& White (1986) to evaluate the surface term used in the estimation of the virial mass of the Coma cluster.

It should be noted that $R_{\mathrm{s}}$ is not the radius of the sphere containing the virialized mass of the sample. An estimation of this type of radius would require knowing the galaxy positions of the sample in time; thus, steady magnitudes could be obtained by a time average. As this is not possible, distances averaged in the sample at present, such as the harmonic radius or the averaged distance between the central galaxy and its companions, would give information about this quantity.

\subsection{Projected mass estimator}

For the $N$ galaxies of the sample addressed in this Sect. 3, the equivalent equation to (28), derived from the continuous model, is

$$
\begin{aligned}
\left\langle R_{\mathrm{S}} S_{\mathrm{vir}}\left(R_{\mathrm{s}}\right)\right\rangle_{t}= & \frac{32}{\pi} \frac{(2-\beta)}{(4-3 \beta)}\left\langle\sum_{i=1}^{N}\left[m_{\mathrm{g}}\right]_{i}\left[r_{i}\right]_{\perp}\left[v_{i}\right]_{\mathrm{Z}}^{2}\right\rangle_{t} \\
& -G\left\langle\sum_{i=1}^{N}\left[m_{\mathrm{g}}\right]_{i}[\mathcal{M}]_{i}\right\rangle_{t}+\frac{128 G \rho_{\Lambda}}{9 c^{2}}\left\langle\sum_{i=1}^{N}\left[m_{\mathrm{g}}\right]_{i}\left[r_{i}\right]_{\perp}^{3}\right\rangle .
\end{aligned}
$$

Thus, by assuming Eqs. (34)-(36), and taking into account that $r_{i=1}=0$ and $[\mathcal{M}]_{1}=0$, Eq. (56) can be read as

$$
\begin{aligned}
R_{\mathrm{s}} S_{\mathrm{vir}}\left(R_{\mathrm{S}}\right)= & \frac{32}{\pi} \frac{(2-\beta)}{(4-3 \beta)} M_{\mathrm{g}}^{c}\left[R_{\perp} \sigma_{\mathrm{z}}^{2}\right] \\
& -G M M_{\mathrm{g}}^{c}[\alpha]+\frac{128 G \rho_{\Lambda}}{9 c^{2}} M_{\mathrm{g}}^{c}\left[R_{\perp}^{3}\right],
\end{aligned}
$$

where

$$
\begin{aligned}
{\left[R_{\perp} \sigma_{\mathrm{z}}^{2}\right] } & \approx\left[R_{\perp} \sigma_{\mathrm{los}}^{2}\right] \\
& =\frac{1}{N-1} \sum_{i=2}^{N}\left[r_{i}\right]_{\perp}\left[\left[V_{i}\right]_{\mathrm{los}}-\frac{1}{N} \sum_{j=1}^{N}\left[V_{j}\right]_{\mathrm{los}}\right]^{2}, \\
{[\alpha] } & =\frac{1}{N-1} \sum_{i=2}^{N} \frac{[\mathcal{M}]_{i}}{M} \\
{\left[R_{\perp}^{3}\right] } & =\frac{1}{N-1} \sum_{i=2}^{N}\left[r_{i}\right]_{\perp}^{3} .
\end{aligned}
$$

As can be seen, the kinetic term of Eq. (56) has been approximated by the kinetic term of Eq. (57). Besides, in Eq. (57) we have assumed $\left[R_{\perp} \sigma_{\mathrm{z}}^{2}\right] \approx\left[R_{\perp} \sigma_{\text {los }}^{2}\right]$; it should be noted that both quantities are not equal, but coincide in the isotropy case (see Eqs. (25) and (26)).

From Eq. (59), the quantity $M[\alpha]$ is the average of $[\mathcal{M}]_{i}$ in the $N-1$ companions to the main galaxy. Thus, for models PM, MG, and EM, we obtain (see Eqs. (42)-(46))

$[\alpha] \equiv[\alpha]_{\mathrm{PM}}=1$,

$[\alpha] \equiv[\alpha]_{\mathrm{MG}}=\frac{\left[\mathcal{M}_{\mathrm{MG}}\right]}{M}=\left[\frac{\left[m_{\mathrm{g}}\right]_{1}}{M_{\mathrm{g}}}+\frac{1}{2} \frac{(N-2)}{(N-1)} \frac{M_{\mathrm{g}}^{c}}{M_{\mathrm{g}}}\right]$,

$[\alpha] \equiv[\alpha]_{\mathrm{EM}}=\frac{\left[\mathcal{M}_{\mathrm{EM}}\right]}{M}=\frac{1}{2}$.

As can be seen, when the main galaxy dominates the group mass, $[\alpha]_{\mathrm{MG}}$ tends to $[\alpha]_{\mathrm{PM}}$; and if all galaxies have the same mass, $[\alpha]_{\mathrm{MG}}=[\alpha]_{\mathrm{EM}}$. When Eq. (61) is used, the gravitational term of Eq. (57) is that derived for $N-1$ galaxies orbiting around a point mass. In the case of using Eq. (63), this gravitational term corresponds to $N$ galaxies with the same mass.

If the surface term of Eq. (57) is neglected, the projected mass estimator is obtained:

$\left\{M_{\text {proj }}\right\}_{\beta}=\left[M_{\text {proj }}\right]_{\beta}\left(1+\left[f_{\text {proj }}\right]_{\beta}\right)$,

where

$$
\begin{aligned}
{\left[M_{\mathrm{proj}}\right]_{\beta} } & =\frac{32}{\pi G} \frac{(2-\beta)}{(4-3 \beta)} \frac{\left[R_{\perp} \sigma_{\mathrm{los}}^{2}\right]}{[\alpha]}, \\
{\left[f_{\mathrm{proj}}\right]_{\beta} } & =\frac{4 \pi G \rho_{\Lambda}}{9 c^{2}} \frac{(4-3 \beta)}{(2-\beta)} \frac{\left[R_{\perp}^{3}\right]}{\left[R_{\perp} \sigma_{\mathrm{los}}^{2}\right]} \\
& =\frac{1}{6} H_{0}^{2} \Omega_{\Lambda} \frac{(4-3 \beta)}{(2-\beta)} \frac{\left[R_{\perp}^{3}\right]}{\left[R_{\perp} \sigma_{\mathrm{los}}^{2}\right]} .
\end{aligned}
$$


Projected mass estimations grow with the anisotropic parameter $\beta$, while the contributions of cosmological constant terms decrease with $\beta$. This can be seen from Eqs. (64)-(66); i.e.

$$
\begin{aligned}
\left\{M_{\text {proj }}\right\}_{\beta} & =\left[3 \frac{(2-\beta)}{(4-3 \beta)}+\left[f_{\text {proj }}\right]_{\beta=-\infty}\right]\left[\frac{\left\{M_{\text {proj }}\right\}_{\beta=-\infty}}{1+\left[f_{\text {proj }}\right]_{\beta=-\infty}}\right], \\
{\left[f_{\text {proj }}\right]_{\beta} } & =\frac{1}{3} \frac{(4-3 \beta)}{(2-\beta)}\left[f_{\text {proj }}\right]_{\beta=-\infty} .
\end{aligned}
$$

The leader term of the mass estimator (64), $\left[M_{\text {proj }}\right]_{\beta}$, depends on $\left[R_{\perp} \sigma_{\mathrm{z}}^{2}\right]$. This kind of dependence was proposed by Page (1952) for estimating the mass of binary galaxies. The Page estimator was based on the mean value of the projected mass and on the assumption of circular orbits $(\beta \rightarrow-\infty)$ for galaxies. Wolf \& Bahcall (1972) also derived a similar expression assuming straight-line orbits $(\beta=1)$. Bahcall \& Tremaine (1981) derived another mass estimator also based on projected mass that depended on the mean value of the square of the Keplerian orbit eccentricities, $e$; their estimator, which does not take into account the cosmological constant contribution, is that of Eq. (65) with $\left\langle e^{2}\right\rangle=(2-\beta)^{-1}$. The mass estimator by Bahcall \& Tremaine (1981) has been used, as an example, by Karachentsev (2005) to estimate the mass of the nearest groups of galaxies; he adopted $\left\langle e^{2}\right\rangle^{1 / 2}=0.7$ as the average eccentricity. This value is close to that for isotropic orbits, $\beta=0$. Heisler et al. (1985) showed that when the distribution of orbit eccentricities is unknown, the assumption of isotropic orbits resulted in a good agreement with the numerical experiments that they carried out.

\subsection{Sample mass estimator}

Using Eqs. (51)-(53) in (37), and Eqs. (64)-(66) in (57), the equations for the mass contained in the sample field or sample mass, $M \equiv\left\{M_{\mathrm{s}}\right\}_{\beta}$, can be derived. Thus,

$$
\begin{aligned}
S_{\mathrm{vir}}\left(R_{\mathrm{s}}\right) & =\frac{G M_{\mathrm{g}}^{c}}{\left[R_{\mathrm{H}}\right]}\left[\left\{M_{\mathrm{vir}}\right\}-\left\{M_{\mathrm{s}}\right\}_{\beta}\right], \\
R_{\mathrm{s}} S_{\mathrm{vir}}\left(R_{\mathrm{s}}\right) & =G M_{\mathrm{g}}^{c}[\alpha]\left[\left\{M_{\mathrm{proj}}\right\}_{\beta}-\left\{M_{\mathrm{s}}\right\}_{\beta}\right] .
\end{aligned}
$$

As in this work, galaxy groups are assumed to be isolated, the surface terms of Eqs. (69) and (70) must be greater than or equal to zero. Hence,

$$
\left\{M_{\mathrm{s}}\right\}_{\beta} \leq \min \left[\left\{M_{\mathrm{vir}}\right\},\left\{M_{\mathrm{proj}}\right\}_{\beta}\right] .
$$

Besides, the sample mass must be greater than or equal to the total mass of the $N$ galaxies; i.e.

$M_{\mathrm{g}} \leq\left\{M_{\mathrm{s}}\right\}_{\beta}$.

The mass estimator for the sample, $\left\{M_{\mathrm{s}}\right\}_{\beta}$, can be obtained from Eqs. (69) and (70). Thus,

$\left\{M_{\mathrm{s}}\right\}_{\beta}=\frac{\left\{M_{\mathrm{vir}}\right\}-[\gamma]\left\{M_{\mathrm{proj}}\right\}_{\beta}}{1-[\gamma]}$,

where

$[\gamma]=[\alpha] \frac{\left[R_{\mathrm{H}}\right]}{R_{\mathrm{S}}}$.

When $[\gamma]<1$, the smallest value of $\left\{M_{\mathrm{s}}\right\}_{\beta}$ is derived for $\beta=1$ (the greatest value of $\left\{M_{\text {proj }}\right\}_{\beta}$ is reached for radial orbits; see Eq. (67)). If $M_{\mathrm{g}} \leq\left\{M_{\mathrm{s}}\right\}_{\beta=1}$, the upper limit for the anisotropy parameter is $\beta_{\mathrm{M}}=1$. But when $M_{\mathrm{g}}>\left\{M_{\mathrm{s}}\right\}_{\beta=1}$, the upper limit $\beta_{\mathrm{M}}<1$ is imposed by $M_{\mathrm{g}}=\left\{M_{\mathrm{s}}\right\}_{\beta_{\mathrm{M}}}$.

Following with the case $[\gamma]<1$, the greatest $\left\{M_{\mathrm{s}}\right\}_{\beta}$ appears for $\beta=-\infty$ (circular orbits lead to the smallest value of $\left\{M_{\mathrm{proj}}\right\}_{\beta}$; see Eq. (67)). When $\left\{M_{\mathrm{s}}\right\}_{\beta=-\infty} \leq \min \left[\left\{M_{\mathrm{vir}}\right\},\left\{M_{\mathrm{proj}}\right\}_{\beta=-\infty}\right]$, $\beta_{\mathrm{m}}=-\infty$ is the lower limit for $\beta$. However, if it happens that $\left\{M_{\mathrm{s}}\right\}_{\beta=-\infty}>\min \left[\left\{M_{\mathrm{vir}}\right\},\left\{M_{\mathrm{proj}}\right\}_{\beta=-\infty}\right]$, the surface terms of Eqs. (69) and (70) are negative; then, $\beta_{\mathrm{m}}$ is calculated from the condition $\left\{M_{\mathrm{s}}\right\}_{\beta_{\mathrm{m}}}=\left\{M_{\mathrm{vir}}\right\}=\left\{M_{\text {proj }}\right\}_{\beta_{\mathrm{m}}}$.

Hence, if $[\gamma]<1$ is fulfilled by a galaxy group,

$M_{\mathrm{g}} \leq\left\{M_{\mathrm{s}}\right\}_{\beta} \leq\left\{M_{\text {vir }}\right\} \leq\left\{M_{\text {proj }}\right\}_{\beta}, \quad \beta_{\mathrm{m}} \leq \beta \leq \beta_{\mathrm{M}}$.

In the case of $[\gamma]>1, \beta_{\mathrm{M}}<1$ appears when $\left\{M_{\mathrm{s}}\right\}_{\beta=1}>$ $\min \left[\left\{M_{\mathrm{vir}}\right\},\left\{M_{\mathrm{proj}}\right\}_{\beta=1}\right]$ (the greater value of $\left\{M_{\mathrm{s}}\right\}_{\beta}$ is now for radial orbits): then, $\beta_{\mathrm{M}}$ derives from the condition $\left\{M_{\mathrm{s}}\right\}_{\beta_{\mathrm{M}}}=$ $\left\{M_{\mathrm{vir}}\right\}=\left\{M_{\text {proj }}\right\}_{\beta_{\mathrm{M}}}$. This means that for the allowed values of $\beta$, $\left\{M_{\text {vir }}\right\} \geq\left\{M_{\text {proj }}\right\}_{\beta}$. Finally, $\beta_{\mathrm{m}} \neq-\infty$ if $\left\{M_{\mathrm{s}}\right\}_{\beta=-\infty}<M_{\mathrm{g}}$ (circular orbits lead to the smaller value of $\left.\left\{M_{\mathrm{s}}\right\}_{\beta}\right)$; so, $M_{\mathrm{g}}=\left\{M_{\mathrm{s}}\right\}_{\beta_{\mathrm{m}}}$ imposes the lower limit for $\beta$. Hence, for a $[\gamma]>1$ galaxy group,

$M_{\mathrm{g}} \leq\left\{M_{\mathrm{s}}\right\}_{\beta} \leq\left\{M_{\text {proj }}\right\}_{\beta} \leq\left\{M_{\text {vir }}\right\}, \quad \beta_{\mathrm{m}} \leq \beta \leq \beta_{\mathrm{M}}$.

We know that the extension of the sample coincides with that of the isolated system when the surface terms are zero. Hence, this occurs for $\gamma<1$ galaxy groups whose anisotropy parameter is $\beta_{\mathrm{m}} \neq-\infty$. Such a situation also appears in a galaxy group fulfilling $\gamma>1$ if $\beta=\beta_{\mathrm{M}}<1$.

Finally, the contribution of cosmological constant terms can be determined from

$\left\{M_{\mathrm{s}}\right\}_{\beta}=\left[M_{\mathrm{s}}\right]_{\beta}\left(1+\left[f_{\mathrm{s}}\right]_{\beta}\right)$,

where $\left[M_{\mathrm{s}}\right]_{\beta}$ is the sample mass neglecting cosmological constant terms. $\left[M_{\mathrm{s}}\right]_{\beta}$ fulfills a similar equation to (73), but where $\left\{M_{\text {vir }}\right\}$ and $\left\{M_{\text {proj }}\right\}_{\beta}$ are substituted by $\left[M_{\text {vir }}\right]$ and $\left[M_{\text {proj }}\right]_{\beta}$. From Eqs. (73), (77), (51), and (64), $\left[f_{\mathrm{s}}\right]_{\beta}$ can be expressed as

$\left[f_{\mathrm{s}}\right]_{\beta}=\frac{\left[f_{\mathrm{vir}}\right]-[\eta]_{\beta}\left[f_{\mathrm{proj}}\right]_{\beta}}{1-[\eta]_{\beta}}$

where

$[\eta]_{\beta}=[\gamma] \frac{\left\{M_{\mathrm{proj}}\right\}_{\beta}}{\left\{M_{\mathrm{vir}}\right\}} \frac{\left(1+\left[f_{\mathrm{vir}}\right]\right)}{\left(1+\left[f_{\mathrm{proj}}\right]_{\beta}\right)}$

\section{Mass estimations of some neighbouring groups of galaxies}

In this section, the estimators of mass presented in Sect. 3 are applied to the galaxy groups studied by Karachentsev (2005): the Milky Way (MW), M 31, M 81, NGC 2403, Centaurus A, M 83, IC 342, Maffei, Sculptor Filament \& Canes Venatici I Cloud (physical quantities have been taken from Karachentsev et al. (2004). All these groups have a main galaxy; this allows us to use the mass estimators when taking the main galaxy as the centre of the group. Hereafter, indicative masses of galaxies appearing in Karachentsev et al. (2004) are taken as galaxy masses.

Different sample mass models are explored. Each one of these models is characterized by two parameters: $\left[R_{\mathrm{H}}\right]$ (appearing in the virial mass estimator; see Eq. (51)), and $[\alpha]$ (used in projected mass estimations; see Eq. (64)). In the following, we deal with $\left[R_{\mathrm{H}}\right]_{\mathrm{PM}},\left[R_{\mathrm{H}}\right]_{\mathrm{MG}},\left[R_{\mathrm{H}}\right]_{\mathrm{EM}}$ and $\left[R_{\mathrm{H}}\right]_{\mathrm{K}}$ given by Eqs. (47)-(50); and with $[\alpha]_{\mathrm{MG}}$ and $[\alpha]_{\mathrm{EM}}$ from Eqs. (62), (63). 
Table 1. Some properties of neighbouring galaxy groups.

\begin{tabular}{|c|c|c|c|c|c|c|c|c|c|c|c|}
\hline Parameter & Units & Milky Way & M 31 & M 81 & NGC 2403 & Cen A & M 83 & IC 342 & Maffei & Sculptor & CVn I \\
\hline$N$ & - & 15 & 19 & 25 & 5 & 28 & 14 & 8 & 8 & 6 & 9 \\
\hline$M_{\mathrm{g}}^{\star}$ & $10^{11} M_{\odot}$ & 1.47 & 2.91 & 1.81 & 0.41 & 6.09 & 0.93 & 1.10 & 0.46 & 1.32 & 0.72 \\
\hline$\left[m_{\mathrm{g}}^{\star}\right]_{1}$ & $10^{11} M_{\odot}$ & 1.41 & 2.70 & 1.66 & 0.37 & 5.37 & 0.91 & 1.00 & 0.37 & 1.07 & 0.46 \\
\hline$\left[\sigma_{l}^{2}\right]^{1 / 2}$ & $\mathrm{~km} \mathrm{~s}^{-1}$ & 75.7 & 77.0 & 68.4 & 22.6 & 105.0 & 71.1 & 53.8 & 58.2 & 54.4 & 56.0 \\
\hline$R_{\mathrm{S}}{ }^{\operatorname{los} 1}$ & $\mathrm{Mpc}$ & 0.696 & 0.824 & 0.520 & 0.484 & 0.634 & 0.531 & 0.741 & 0.294 & 0.710 & 0.602 \\
\hline$[R]$ & $\mathrm{Mpc}$ & 0.199 & 0.280 & 0.196 & 0.448 & 0.363 & 0.208 & 0.417 & 0.134 & 0.438 & 0.518 \\
\hline$\left[R_{\perp}\right]$ & $\mathrm{Mpc}$ & 0.156 & 0.220 & 0.154 & 0.352 & 0.285 & 0.163 & 0.328 & 0.105 & 0.344 & 0.407 \\
\hline$\left[R_{\perp}^{2}\right]$ & $\mathrm{Mpc}^{2}$ & 0.052 & 0.092 & 0.040 & 0.131 & 0.101 & 0.038 & 0.145 & 0.015 & 0.122 & 0.178 \\
\hline$\left[R_{\perp}^{\overline{3}}\right]$ & $\mathrm{Mpc}^{3}$ & 0.024 & 0.052 & 0.014 & 0.051 & 0.041 & 0.011 & 0.069 & 0.002 & 0.049 & 0.827 \\
\hline$\left[R_{\perp} \sigma_{\mathrm{los}}^{2}\right]$ & 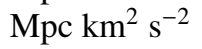 & 819 & 816 & 839 & 197 & 2390 & 818 & 931 & 128 & 1259 & 1283 \\
\hline$\left[R_{\mathrm{H}}\right]_{\mathrm{PM}}$ & $\mathrm{Mpc}$ & 0.107 & 0.065 & 0.088 & 0.507 & 0.292 & 0.140 & 0.123 & 0.117 & 0.522 & 0.583 \\
\hline$\left[R_{\mathrm{H}}\right]_{\mathrm{MG}}$ & $\mathrm{Mpc}$ & 0.110 & 0.068 & 0.092 & 0.542 & 0.311 & 0.142 & 0.130 & 0.132 & 0.588 & 0.735 \\
\hline$\left[R_{\mathrm{H}}\right]_{\mathrm{EM}}$ & $\mathrm{Mpc}$ & 0.214 & 0.130 & 0.176 & 1.014 & 0.584 & 0.280 & 0.246 & 0.234 & 1.044 & 1.166 \\
\hline$\left[R_{\mathrm{H}}\right]_{\mathrm{K}}$ & $\mathrm{Mpc}$ & 0.230 & 0.138 & 0.184 & 1.268 & 0.605 & 0.301 & 0.282 & 0.268 & 1.254 & 1.312 \\
\hline$[\alpha]_{\mathrm{MG}}$ & - & 0.979 & 0.961 & 0.956 & 0.936 & 0.939 & 0.987 & 0.952 & 0.888 & 0.888 & 0.793 \\
\hline$\left[t_{\text {cross }} H_{0}\right]$ & - & 0.187 & 0.258 & 0.204 & 1.408 & 0.245 & 0.208 & 0.550 & 0.164 & 0.572 & 0.656 \\
\hline
\end{tabular}

Some characteristics of the groups are shown in Table 1. The following data are presented in the rows in the table:

1. Number of members in the group, $N$. All members fulfill that their Keplerian cyclic periods are smaller than the timescale of the Universe at present (see Karachentsev 2005).

2. Sum of indicative masses of galaxies in the group, $M_{\mathrm{g}}^{\star}$. Each galaxy indicative mass derives from values of HI rotation velocities and major linear diameters (see Karachentsev et al. 2004). Hereafter, upper index " $\star$ ” is used to indicate indicative mass.

3. Indicative mass of the main galaxy, $\left[m_{\mathrm{g}}^{\star}\right]_{1}$. As can be seen, contributions of $\left[m_{\mathrm{g}}^{\star}\right]_{1}$ to $M_{\mathrm{g}}^{\star}$ are $63 \%$ for $\mathrm{CVn} \mathrm{I}$, and around $80 \%$ for Maffei and Sculptor; from $88 \%$ to $93 \%$ for Centaurus A, NGC 2403, M 81, and M 81; while for the Milky Way and $\mathrm{M} 83$, the mass contributions of main galaxies are greater than $96 \%$.

4. Dispersion of the line-of-sight velocities in the group, $\left[\sigma_{\text {los }}^{2}\right]^{1 / 2}$ (see Eq. (38)). See the small value taken by this magnitude in NGC 2403.

5. Distance of the farthest galaxy from the central massive galaxy of the group, $R_{\mathrm{s}}$.

6. Mean separation among the dominant galaxy and companions to it, $[R]$. This is a measure of the size of the group calculated from the mean projected separation of galaxies from the principal galaxy, $\left[R_{\perp}\right]$, i.e.

$[R]=\frac{4}{\pi}\left[R_{\perp}\right]$,

where

$\left[R_{\perp}\right]=\left[\frac{1}{N-1} \sum_{i=2}^{N}\left[r_{i}\right]_{\perp}\right]$.

(Equation (80) has been derived from the continuous model.)

7. Mean projected separation of galaxies from the principal galaxy, $\left[R_{\perp}\right]$ (see Eq. (81)).

8. Mean square projected separation of galaxies from the principal galaxy, $\left[R_{\perp}^{2}\right]$ (see Eq. (40)).

9. Mean cube projected separation of galaxies from the principal galaxy, $\left[R_{\perp}^{3}\right]$ (see Eq. (60)).
10. $\left[R_{\perp} \sigma_{\text {los }}^{2}\right]$ (see Eq. (58)). We note that this quantity is much smaller for NGC 2403 and Maffei than for the rest of the galaxy groups.

11. Mean harmonic radius assuming that $N-1$ galaxies orbit around a point mass, $\left[R_{\mathrm{H}}\right]_{\mathrm{PM}}$ (see Eq. (47)).

12. Mean harmonic radius given by Eq. (48), $\left[R_{\mathrm{H}}\right]_{\mathrm{MG}}$. The difference between $\left[R_{\mathrm{H}}\right]_{\mathrm{MG}}$ and $\left[R_{\mathrm{H}}\right]_{\mathrm{PM}}$ for a galaxy group is a consequence of the influence of the companions to the main galaxy. The greatest difference is found in the CVn I Cloud: $\left[R_{\mathrm{H}}\right]_{\mathrm{MG}}$ differs from $\left[R_{\mathrm{H}}\right]_{\mathrm{PM}}$ by about $26 \%$. For the Maffei group and for Sculptor Filament, it is $13 \%$. For the rest of the groups, however, it is smaller than $7 \%$. We note that NGC 2403 and CVn I show a $\left[R_{\mathrm{H}}\right]_{\mathrm{MG}}$ greater than $R_{\mathrm{s}}$, and that for the Sculptor group they are comparable.

13. Mean harmonic radius $\left[R_{\mathrm{H}}\right]_{\mathrm{EM}}$. This is twice $\left[R_{\mathrm{H}}\right]_{\mathrm{PM}}$ (see Eq. (49)).

14. Mean harmonic radius in agreement with Karachentsev (2005), $\left[R_{\mathrm{H}}\right]_{\mathrm{K}}$ (see Eq. (50)).

15. Adimensional quantity, $[\alpha]_{\mathrm{MG}}$, given by Eq. (62). This is used in the calculation of $\left\{M_{\text {proj }}\right\}_{\beta}$ and $\left\{M_{\mathrm{s}}\right\}_{\beta}$. This quantity is an average of the fraction of mass inside a sphere covering up to the location of each galaxy. When mass contributions of companions to the main galaxy are negligible, $[\alpha]_{\mathrm{MG}}=1$.

16. Quotient between crossing time, $t_{\text {cross }}$ and timescale of the Universe, $1 / H_{0}$ :

$\left[t_{\text {cross }} H_{0}\right] \approx\left(\frac{[R]}{1 \mathrm{Mpc}}\right)\left(\frac{\left[\sigma_{\mathrm{los}}^{2}\right]^{1 / 2}}{1 \mathrm{~km} \mathrm{~s}^{-1}}\right)^{-1}\left(\frac{H_{0}}{1 \mathrm{~km} \mathrm{~s}^{-1} \mathrm{Mpc}^{-1}}\right)$.

NGC 2403 presents a crossing time greater than the timescale of the Universe at present; this would mean that NGC 2403 is not virialized. The groups IC 342, Sculptor, and $\mathrm{CVn} \mathrm{I}$, show $t_{\text {cross }}$ about half $H_{0}^{-1}$; so, their virialization is not confirmed. The rest of the groups would be in a dynamic equilibrium.

For the Milky Way, to account for projection effects, $\left[R_{\mathrm{H}}\right]_{\mathrm{PM}}$, $\left[R_{\perp}\right],\left[R_{\perp}^{2}\right],\left[R_{\perp}^{3}\right]$ and $\left[R_{\perp} \sigma_{\text {los }}^{2}\right]$ have been calculated as follows:

$\left[R_{\mathrm{H}}\right]_{\mathrm{PM}}=\frac{\pi}{2}\left[\frac{4}{\pi(N-1)} \sum_{i=2}^{N} \frac{1}{\left[D_{i}\right]}\right]^{-1}$, 


$$
\begin{aligned}
& {\left[R_{\perp}\right]=\frac{\pi}{4(N-1)} \sum_{i=2}^{N}\left[D_{i}\right],} \\
& {\left[R_{\perp}^{2}\right]=\frac{2}{3(N-1)} \sum_{i=2}^{N}\left[D_{i}\right]^{2},} \\
& {\left[R_{\perp}^{3}\right]=\frac{3 \pi}{16(N-1)} \sum_{i=2}^{N}\left[D_{i}\right]^{3},} \\
& {\left[R_{\perp} \sigma_{\mathrm{los}}^{2}\right]=\frac{\pi}{4(N-1)} \sum_{i=2}^{N}\left[D_{i}\right]\left[\left[V_{i}\right]_{\mathrm{los}}-\frac{1}{N} \sum_{j=1}^{N}\left[V_{j}\right]_{\mathrm{los}}\right]^{2},}
\end{aligned}
$$

where $\left[D_{i}\right]$ is the distance to the ith galaxy.

\subsection{Selection of harmonic radius model}

In this subsection, we explore harmonic radius models. The differences between them come from the assumption made for the mass enclosed by the sphere covering up to the location of each galaxy, $[\mathcal{M}]_{i}$. One model takes a central point mass, $\left(\right.$ see $\left[\mathcal{M}_{\mathrm{PM}}\right]_{i}$ given by Eq. (42)). Another considers that companions to the main galaxy have the same mass (see $\left[\mathcal{M}_{\mathrm{MG}}\right]_{i}$ given by Eq. (43)). The third model assumes the same mass for all galaxies (see $\left[\mathcal{M}_{\mathrm{EM}}\right]_{i}$ given by Eq. (44)).

In the discrete model, to introduce another average, these masses have been averaged in the galaxy number of each sample. $\left[\mathcal{M}_{\mathrm{PM}}\right],\left[\mathcal{M}_{\mathrm{MG}}\right]$, and $\left[\mathcal{M}_{\mathrm{EM}}\right]$ come from averaging in the $N-1$ companions to the main galaxy; and $\left[\mathcal{M}_{\mathrm{K}}\right]$, from averaging in the $N$ galaxies of the sample. Results for harmonic radii, $\left[R_{\mathrm{H}}\right]_{\mathrm{PM}}$, $\left[R_{\mathrm{H}}\right]_{\mathrm{MG}},\left[R_{\mathrm{H}}\right]_{\mathrm{EM}}$, and $\left[R_{\mathrm{H}}\right]_{\mathrm{K}}$ (see Eqs. (47)-(50)) are shown in rows 11-14 of Table 1 for different galaxy groups.

In this subsection, we select just one harmonic radius model for each group. For this purpose, we come back to the continuous model that assumes power-like mass distributions. These mass distributions would represent $[\mathcal{M}]_{i}$ in the continuous model. They have a power-exponent, $p \geq 0$, and a distribution radius, $R$. The mass enclosed up to $R$ is the sample mass. We impose that discrete and continuous models lead to the same harmonic radius and to the same mean separation between galaxies and the main galaxy. This allows us to fix $p$ and $R$. The criterion we use is to choose the mass distribution of those mentioned, whose distribution radius, $R$, is the closest to the radius at which the farthest galaxy of the sample is located, $R_{\mathrm{S}}$ (see row 5 in Table 1 ).

For the equal mass galaxy model, we assume

$$
\left[\mathcal{M}_{\mathrm{EM}}\right](r)=M\left(\frac{r}{R}\right)^{p} \text {. }
$$

The point mass model is derived from Eq. (88), taking $p=0$; i.e.

$$
\left[\mathcal{M}_{\mathrm{PM}}\right](r)=M \text {. }
$$

For the case of companions to the main galaxy of equal mass, we use

$$
\left[\mathcal{M}_{\mathrm{MG}}\right](r)=\frac{M}{M_{\mathrm{g}}}\left[\left[m_{\mathrm{g}}\right]_{1}+\left[m_{\mathrm{g}}\right]^{c}(r)\right]
$$

In Eq. (90), $\left[m_{\mathrm{g}}\right]_{1}$ corresponds to the main galaxy mass of the sample; and $\left[m_{\mathrm{g}}\right]^{c}(r)$ is the mass distribution of companions to it, given by

$\left[m_{\mathrm{g}}\right]^{c}(r)=\frac{M_{\mathrm{g}}^{c}}{R^{p}-R_{1}^{p}}\left(r^{p}-R_{1}^{p}\right), \quad r \geq R_{1}$,

$\left[m_{\mathrm{g}}\right]^{c}(r)=0, \quad 0 \leq r<R_{1}$ (as can be seen, at $r<R_{1}$, there is no companion to the main galaxy).

The denominator of Eq. (91) has been chosen in order for

$M_{\mathrm{g}}^{c}=\int_{\left[m_{\mathrm{g}}\right]^{c}\left(R_{1}\right)}^{\left[m_{\mathrm{g}}\right]^{c}(R)} \mathrm{d}\left[m_{\mathrm{g}}\right]^{c}$

to be fulfilled. In Eq. (92) (see Eq. (91)),

$\mathrm{d}\left[m_{\mathrm{g}}\right]^{c}=\frac{M_{\mathrm{g}}^{c}}{R^{p}-R_{1}^{p}} p r^{p-1} \mathrm{~d} r, \quad r \geq R_{1}$,

$\mathrm{d}\left[m_{\mathrm{g}}\right]^{c}=0, \quad 0 \leq r<R_{1}$.

The numerator of Eq. (91) makes Eq. (90) equal to Eq. (88) at any $r \geq R_{1}$ when the same mass is assumed for all galaxies. This can be seen when taking into account that, in this case,

$\frac{M}{M_{\mathrm{g}}}\left[m_{\mathrm{g}}\right]_{1}=\left[\mathcal{M}_{\mathrm{EM}}\right]\left(R_{1}\right)=M\left(\frac{R_{1}}{R}\right)^{p}$,

$\frac{M}{M_{\mathrm{g}}} M_{\mathrm{g}}^{c}=\left[\mathcal{M}_{\mathrm{EM}}\right](R)-\left[\mathcal{M}_{\mathrm{EM}}\right]\left(R_{1}\right)=M \frac{R^{p}-R_{1}^{p}}{R^{p}}$.

Thus, using Eqs. (94) and (95) in Eq. (90), Eq. (88) is obtained.

In the continuous model, the equivalent expression to Eq. (39) is

$\frac{1}{\left[R_{\mathrm{H}}\right]}=\frac{1}{M M_{\mathrm{g}}^{c}} \int_{\left[m_{\mathrm{g}}\right]^{c}\left(R_{1}\right)}^{\left[m_{\mathrm{g}}\right]^{c}(R)} \frac{[\mathcal{M}]}{r} \mathrm{~d}\left[m_{\mathrm{g}}\right]^{c}$.

Hence, assuming that $R_{1} \ll R$,

$\left[R_{\mathrm{H}}\right]_{\mathrm{PM}}=\frac{p-1}{p} R$

$\left[R_{\mathrm{H}}\right]_{\mathrm{MG}}=\frac{M_{\mathrm{g}}}{\left[m_{\mathrm{g}}\right]_{1}} \frac{p-1}{p}\left[1+\frac{M_{\mathrm{g}}^{c}}{\left[m_{\mathrm{g}}\right]_{1}} \frac{(p-1)}{(2 p-1)}\right]^{-1} R$,

$\left[R_{\mathrm{H}}\right]_{\mathrm{EM}}=\frac{2 p-1}{p} R$.

To derive Eqs. (97)-(99), Eqs. (89), (90), and (88), respectively, have been used in Eq. (96). As can be seen, when $M_{\mathrm{g}}^{c} \ll\left[m_{\mathrm{g}}\right]_{1}$, $\left[R_{\mathrm{H}}\right]_{\mathrm{MG}} \rightarrow\left[R_{\mathrm{H}}\right]_{\mathrm{PM}}$; and, when Eqs. (94) and (95) are assumed (i.e., when it is assumed that all galaxies have the same mass), $\left[R_{\mathrm{H}}\right]_{\mathrm{MG}} \rightarrow\left[R_{\mathrm{H}}\right]_{\mathrm{EM}}$.

The equivalent equation to the mean separation of galaxies from the main galaxy, $[R]$, given by Eq. (80) is

$[R]=\frac{1}{M_{\mathrm{g}}^{c}} \int_{\left[m_{\mathrm{g}}\right]^{c}\left(R_{1}\right)}^{\left[m_{\mathrm{g}}\right]^{c}(R)} r \mathrm{~d}\left[m_{\mathrm{g}}\right]^{c}$.

So, for $R_{1} \ll R$,

$[R]=\frac{p}{p+1} R$.

Therefore, using Eqs. (97)-(99) in Eq. (101), $p_{\mathrm{PM}}, p_{\mathrm{MG}}$, and $p_{\mathrm{EM}}$ can be derived from

$$
\begin{aligned}
& p_{\mathrm{PM}}=\left[1-\frac{\left[R_{\mathrm{H}}\right]_{\mathrm{PM}}}{[R]}\right]^{-1 / 2}, \\
& p_{\mathrm{MG}}^{2}\left\{1-\frac{\left[m_{\mathrm{g}}\right]_{1}}{M_{\mathrm{g}}}\left[1+\frac{M_{\mathrm{g}}^{c}}{\left[m_{\mathrm{g}}\right]_{1}} \frac{\left(p_{M G}-1\right)}{\left(2 p_{M G}-1\right)}\right] \frac{\left[R_{\mathrm{H}}\right]_{\mathrm{MG}}}{[R]}\right\}=1, \\
& p_{\mathrm{EM}}=\frac{1}{2}\left[-1+\sqrt{1+4\left(2-\frac{\left[R_{\mathrm{H}}\right]_{\mathrm{EM}}}{[R]}\right)}\right]\left[2-\frac{\left[R_{\mathrm{H}}\right]_{\mathrm{EM}}}{[R]}\right]^{-1} .
\end{aligned}
$$


Table 2. Power index, $p$, and distribution radius, $R$, of power-like mass distributions representing $\left[\mathcal{M}_{\mathrm{PM}}\right],\left[\mathcal{M}_{\mathrm{MG}}\right],\left[\mathcal{M}_{\mathrm{EM}}\right]$, and $\left[\mathcal{M}_{\mathrm{K}}\right]$ in the continuous model.

\begin{tabular}{cllllrrrrrrr}
\hline \hline Parameter & Units & MW & M 31 & M 81 & N2403 & Cen A & M 83 & I342 & Maffei & Sculptor & CVn I \\
\hline$p_{\mathrm{PM}}$ & - & 1.471 & 1.141 & 1.347 & - & 2.261 & 1.749 & 1.191 & 0.281 & - & - \\
$p_{\mathrm{MG}}$ & - & 1.468 & 1.137 & 1.328 & - & 2.190 & 1.745 & 1.186 & 0.274 & - & - \\
$p_{\mathrm{EM}}$ & - & 0.631 & 0.545 & 0.601 & - & 0.769 & 0.689 & 0.559 & 0.827 & - & - \\
$p_{\mathrm{K}}$ & - & 0.647 & 0.548 & 0.608 & - & 0.791 & 0.716 & 0.570 & - & - & - \\
$R_{\mathrm{PM}}$ & $\mathrm{Mpc}$ & 0.334 & 0.525 & 0.342 & - & 0.524 & 0.327 & 0.767 & 0.182 & - & - \\
$R_{\mathrm{MG}}$ & $\mathrm{Mpc}$ & 0.335 & 0.526 & 0.344 & - & 0.529 & 0.327 & 0.769 & 0.183 & - & - \\
$R_{\mathrm{EM}}$ & $\mathrm{Mpc}$ & 0.514 & 0.794 & 0.522 & - & 0.835 & 0.510 & 1.163 & 0.296 & - & - \\
$R_{\mathrm{K}}$ & $\mathrm{Mpc}$ & 0.507 & 0.791 & 0.518 & - & 0.822 & 0.498 & 1.149 & - & - & - \\
$R_{\mathrm{S}}$ & $\mathrm{Mpc}$ & 0.696 & 0.824 & 0.520 & 0.484 & 0.634 & 0.531 & 0.741 & 0.294 & 0.710 & 0.602 \\
\hline
\end{tabular}

Notes. For each group, the distance $R_{\mathrm{s}}$ of the farthest galaxy (from the main galaxy) is also presented.

For each group, values of $M_{\mathrm{g}}^{\star},\left[m_{\mathrm{g}}^{\star}\right]_{1}, M_{\mathrm{g}}^{c}=M_{\mathrm{g}}^{\star}-\left[m_{\mathrm{g}}^{\star}\right]_{1}$, $[R],\left[R_{\mathrm{H}}\right]_{\mathrm{PM}},\left[R_{\mathrm{H}}\right]_{\mathrm{MG}},\left[R_{\mathrm{H}}\right]_{\mathrm{EM}}$, and $\left[R_{\mathrm{H}}\right]_{\mathrm{K}}$ have been taken from Table 1. Thus, Eq. (102), for $\left[R_{\mathrm{H}}\right]_{\mathrm{PM}}$, allows us to know $p_{\mathrm{PM}}$; $p_{\mathrm{MG}}$ is obtained by solving Eq. (103) for $\left[R_{\mathrm{H}}\right]_{\mathrm{MG}}$; and Eq. (104), for $\left[R_{\mathrm{H}}\right]_{\mathrm{EM}}$, and $\left[R_{\mathrm{H}}\right]_{\mathrm{K}}$, leads to $p_{\mathrm{EM}}$ and $p_{\mathrm{K}}$, respectively. Then, $R_{\mathrm{PM}}, R_{\mathrm{MG}}, R_{\mathrm{EM}}$, and $R_{\mathrm{K}}$ are derived from Eq. (101). The results are shown in Table 2. The last row in this table again shows $R_{\mathrm{S}}$ (see row 5 in Table 1 ) to be compared with $R_{\mathrm{PM}}, R_{\mathrm{MG}}, R_{\mathrm{EM}}$, and $R_{\mathrm{K}}$ in each group.

From Table 2, it can be seen that NGC 2403, Sculptor Filament, and Canes Venatici I Cloud have no solution. It should be remembered that NGC 2403 is a non-virialized group and the other two groups have a dubious virialization.

As can be seen from Table 2, for the Milky Way, M 31, M 81 and $\mathrm{M} 83$ groups, $\left[R_{\mathrm{H}}\right]_{\mathrm{EM}}$ and $\left[R_{\mathrm{H}}\right]_{\mathrm{K}}$ lead to distribution radii that are closer to their $R_{\mathrm{S}}$ than those derived from $\left[R_{\mathrm{H}}\right]_{\mathrm{PM}}$ and $\left[R_{\mathrm{H}}\right]_{\mathrm{MG}}$. We can see that for these groups, $\left[m_{\mathrm{g}}^{\star}\right]_{1} / M_{\mathrm{g}}^{\star} \approx 0.96,0.93,0.92$, and 0.98 , respectively. In spite of these large contributions of the main galaxy mass to the group galaxy mass, these groups are better represented by a mass distribution of equal mass galaxies. The reason has to be found in the locations of galaxies with respect to the main galaxy of each group.

The opposite behaviour occurs with Centaurus A and IC 342. These groups show smaller values of $\left[m_{\mathrm{g}}^{\star}\right]_{1} / M_{\mathrm{g}}^{\star}(0.88$ and 0.91$)$. However, they are better represented by mass distributions, assuming a mass dominant galaxy; so, $\left[R_{\mathrm{H}}\right]_{\mathrm{PM}}$ and $\left[R_{\mathrm{H}}\right]_{\mathrm{MG}}$ are better models for these galaxy groups.

A different case is Maffei. For this group, $\left[m_{\mathrm{g}}^{\star}\right]_{1} / M_{\mathrm{g}}^{\star} \approx 0.80$. We have found that the best mass distribution of those studied is that which assumes the same mass for all galaxies; i.e. the harmonic radius leading to the best fitting to $R_{\mathrm{S}}$ is $\left[R_{\mathrm{H}}\right]_{\mathrm{EM}}$. We note that Maffei does not have a solution for $\left[R_{\mathrm{H}}\right]_{\mathrm{K}}$.

It is noticeable that the worst fittings are for the Milky Way ( $R$ differs from $R_{\mathrm{S}}$ by about $26 \%$ ) and for Centaurus A (17\%). For the rest of the groups $\left|R_{\mathrm{s}}-R\right| / R_{\mathrm{s}}<4 \%$.

From the above results, and according to the criterion proposed in this subsection, we have thought to take $\left[R_{\mathrm{H}}\right]_{\mathrm{EM}}$ for the Milky Way, M 31, M 81, M 83, and Maffei; and $\left[R_{\mathrm{H}}\right]_{\mathrm{MG}}$ for Centaurus A and IC 342. As we have not found any solution for NGC 2403, Sculptor, and CVn I, we will deal with both $\left[R_{\mathrm{H}}\right]_{\mathrm{MG}}$ and $\left[R_{\mathrm{H}}\right]_{\mathrm{EM}}$.

\subsection{Mass estimations neglecting surface effects}

In this subsection, we estimate virial and projected masses for neighbouring galaxy groups. Virial masses are derived from Eq. (51), assuming the harmonic radii chosen in the previous subsection (see Tables 1 and 2). Projected masses are obtained from Eq. (64) using $[\alpha]_{\mathrm{MG}}$ (see Eq. (62) and Table 1) and $[\alpha]_{\mathrm{EM}}=1 / 2$ (see Eq. (63)). Results are shown in Tables 3-5.

Table 3 is devoted to the Milky Way, M31, M 81, M 83, and Maffei groups; these groups fulfill that $\left[R_{\mathrm{H}}\right]_{\mathrm{EM}}$ is the best harmonic radius of those considered according to the criterion proposed in the previous subsection. Table 4 presents the results for Centaurus A and IC 342, for which, $\left[R_{\mathrm{H}}\right]_{\mathrm{MG}}$ is better than the other harmonic radii. And, Table 5 shows NGC 2403, Sculptor, and CVn I results, assuming both $\left[R_{\mathrm{H}}\right]_{\mathrm{MG}}$ and $\left[R_{\mathrm{H}}\right]_{\mathrm{EM}}$ (the criterion given in the previous subsection is not applicable).

Estimations of the virial masses are presented in the first panels of Tables 3-5. In the second and third panels, the projected masses for circular orbits, isotropy, and radial orbits are shown. In these panels, the growing behaviour of $\left\{M_{\text {proj }}\right\}_{\beta}$ with respect to $\beta$ (see Eq. (67)), can be appreciated. $[\alpha]_{\mathrm{MG}}$ and $[\alpha]_{\mathrm{EM}}$ are assumed in the second and third panels, respectively.

The virial and projected masses shown in Tables 3-5 differ from those given by Karachentsev (2005). This is because Karachentsev took $\left[R_{\mathrm{H}}\right]=\left[R_{\mathrm{H}}\right]_{\mathrm{K}}$ (see Eq. (50) and Table 1), $[\alpha]=[\alpha]_{\mathrm{PM}}=1$ (see Eq. $(61)$ ), and $\left\langle e^{2}\right\rangle^{1 / 2}=0.7(\beta \approx 0)$. His results can be compared with those of our models assuming $\left[R_{\mathrm{H}}\right]=\left[R_{\mathrm{H}}\right]_{\mathrm{EM}},[\alpha]=[\alpha]_{\mathrm{MG}}$ and $\beta=0$. Nevertheless, we have seen that, according to the criterion used (see Sect. 4.1), these models are not suitable for Centaurus A and IC 342. The results are similar for the Milky Way, M31, and M 83. The virial masses are comparable for Maffei; however, the projected masses differ by about ten times (this is most probably due to a mistake in the writing; in fact, in Karachentsev et al. (2003c), both projected masses are similar). With respect to the probably non-virialized groups addressed by us, the projected masses are comparable for Sculptor Filament but the virial masses differ appreciably. The virial masses are similar for Canes Venatici I Cloud, but the projected masses are different. We note that, in the work by Karachentsev (2005), M 81 and NGC 2403 are studied only as a single group. The virial and projected masses for M 81 NGC 2403 complex will be addressed in Sect. 5.2 (see Table 14).

When reading Tables $3-5$, we must bear in mind that the sample mass must be smaller than or equal to the minimum of virial and projected mass estimations (the groups are considered isolated).

We note that Maffei shows $\left\{M_{\text {proj }}\right\}_{\beta}$ rather smaller than $\left\{M_{\text {vir }}\right\}$ when $[\alpha]_{\mathrm{MG}}$ is taken. In the case of assuming $[\alpha]_{\mathrm{EM}}$, only for $\beta>0.950$, the projected masses are greater than the virial mass. This is due to the small value of $\left[R_{\perp} \sigma_{\text {los }}^{2}\right]$. It is interesting to compare $\left[R_{\mathrm{H}}\right]_{\mathrm{EM}}\left[\sigma_{\text {los }}^{2}\right]$ with $\left[R_{\perp} \sigma_{\text {los }}^{2}\right]$. The former, which contributes 
Table 3. Virial and projected mass estimations (in units of $10^{11} M_{\odot}$ ) for the Milky Way, M 31, M 81, M 83, and Maffei groups.

\begin{tabular}{clrrrrr}
\hline \hline Model & Parameter & MW & M 31 & M 81 & M 83 & Maffei \\
\hline$\left[R_{\mathrm{H}}\right]_{\mathrm{EM}}$ & $\left\{M_{\text {vir }}\right\}$ & 8.73 & 5.57 & 9.29 & 10.01 & 5.58 \\
\hline$[\alpha]_{\text {MG }}$ & $\left\{M_{\text {proj }}\right\}_{\beta=-\infty}$ & 6.96 & 7.49 & 7.13 & 6.70 & 1.17 \\
{$[\alpha]_{\text {MG }}$} & $\left\{M_{\text {proj }}\right\}_{\beta=0}$ & 10.26 & 10.84 & 10.59 & 9.97 & 1.74 \\
{$[\alpha]_{\text {MG }}$} & $\left\{M_{\text {proj }}\right\}_{\beta=1}$ & 20.17 & 20.91 & 20.98 & 19.78 & 3.44 \\
\hline$[\alpha]_{\text {EM }}$ & $\left\{M_{\text {proj }}\right\}_{\beta=-\infty}$ & 13.62 & 14.39 & 13.63 & 13.22 & 2.06 \\
{$[\alpha]_{\text {EM }}$} & $\left\{M_{\text {proj }}\right\}_{\beta=0}$ & 20.08 & 20.83 & 20.25 & 19.67 & 3.09 \\
{$[\alpha]_{\text {EM }}$} & $\left\{M_{\text {proj }}\right\}_{\beta=1}$ & 39.47 & 40.16 & 40.12 & 39.05 & 6.11 \\
\hline & {$\left[f_{\text {vir }}\right]_{1}$} & 0.02 & 0.03 & 0.01 & 0.01 & 0.01 \\
& {$\left[f_{\text {proj }}\right]_{\beta=-\infty}$} & 0.05 & 0.12 & 0.03 & 0.02 & 0.04 \\
& {$\left[f_{\text {proj }}\right]_{\beta=0}$} & 0.04 & 0.08 & 0.02 & 0.02 & 0.02 \\
& {$\left[f_{\text {proj }}\right]_{\beta=1}$} & 0.02 & 0.04 & 0.01 & 0.01 & 0.01 \\
\hline
\end{tabular}

Notes. Contributions of cosmological constant terms to these masses are also shown.

to $\left\{M_{\mathrm{vir}}\right\}$, is much greater than the latter, which contributes to $\left\{M_{\text {proj }}\right\}_{\beta}$ (note that $\left[R_{\mathrm{H}}\right]_{\mathrm{EM}}\left[\sigma_{\text {los }}^{2}\right] \approx 6.2\left[R_{\perp} \sigma_{\text {los }}^{2}\right]$ ).

The contributions of cosmological constant terms to virial and projected mass estimators are shown in the fourth panels of Tables 3-5. In these panels, the decreasing behaviour of $\left[f_{\text {proj }}\right]_{\beta}$ with $\beta$ (see Eq. (68)) can be seen. According to $\left[f_{\text {vir }}\right]$, the studied groups can be classified as those with $\left[f_{\text {vir }}\right] \leq 0.03$ (Milky Way, M 31, M 81, M 83, Maffei, and Centaurus A), and those with $\left[f_{\text {vir }}\right] \geq 0.08$ (IC 342, Sculptor, CVn I, and NGC 2403, which shows the greatest value, $\left[f_{\text {vir }}\right] \approx 0.5$ ). We note that this last set of galaxy groups presents $\left[t_{\text {cross }} H_{0}\right]>0.5$ (questionable virialization) and overcoat NGC 2403 that fulfilling $\left[t_{\text {cross }} H_{0}\right]=$ 1.4 , it is a non-virialized group. These results lead us to think about the possibility that non-virialized groups present values of $\left[R_{\perp}^{2}\right] /\left[\sigma_{\text {los }}^{2}\right]$ that are much greater than those presented by virialized groups. For the studied groups with questionable virialization, $\left[R_{\perp}^{2}\right] /\left[\sigma_{\mathrm{los}}^{2}\right]>4 \times 10^{-5} \mathrm{Mpc}^{2} \mathrm{~km}^{-2} \mathrm{~s}^{2}$ (for NGC 2403, $\left[R_{\perp}^{2}\right] /\left[\sigma_{\text {los }}^{2}\right] \approx 2.6 \times 10^{-4} \mathrm{Mpc}^{2} \mathrm{~km}^{-2} \mathrm{~s}^{2}$ ), while for the other groups, $\left[R_{\perp}^{2}\right] /\left[\sigma_{\text {los }}^{2}\right]<1.5 \times 10^{-5} \mathrm{Mpc}^{2} \mathrm{~km}^{-2} \mathrm{~s}^{2}$.

Chernin et al. (2012), by taking $1 \mathrm{Mpc}$ and $70 \mathrm{~km} \mathrm{~s}^{-1}$ as the characteristic size and velocity of galaxy groups, estimated that the contribution of the cosmological constant term to the virial mass estimation is about $f_{\mathrm{C}} \approx 0.8$ (see Eq. (54)). Comparing the leader terms of Eqs. (51) and (54), we have that $v^{2} \approx 3\left[\sigma_{\text {los }}^{2}\right]$, and $r \approx\left[R_{\mathrm{H}}\right]$. Taking values from Table 1, NGC 2403 presents $f_{\mathrm{C}} \equiv\left[f_{\mathrm{C}}\right]_{\mathrm{MG}}=(1 / 3) H_{0}^{2} \Omega_{\Lambda}\left[R_{\mathrm{H}}\right]_{\mathrm{MG}}^{2} /\left[\sigma_{\text {los }}^{2}\right] \approx 0.7$ and $f_{\mathrm{C}} \equiv\left[f_{\mathrm{C}}\right]_{\mathrm{EM}}=(1 / 3) H_{0}^{2} \Omega_{\Lambda}\left[R_{\mathrm{H}}\right]_{\mathrm{EM}}^{2} /\left[\sigma_{\text {los }}^{2}\right] \approx 2.5$; Sculptor and $\mathrm{CVn}$ I show about $\left[f_{\mathrm{C}}\right]_{\mathrm{MG}} \approx 0.2$ and $\left[f_{\mathrm{C}}\right]_{\mathrm{EM}} \approx 0.5$; , while for the rest of the groups, $\left[f_{\mathrm{C}}\right]_{\mathrm{MG}}<0.01$ and $\left[f_{\mathrm{C}}\right]_{\mathrm{EM}}<0.02$. Moreover, when comparing the leader terms and cosmological constant terms of Eqs. (51) and (54), we see that $\left[f_{\text {vir }}\right] \approx(3 / 2)\left(\left[R_{\perp}^{2}\right] /\left[R_{\mathrm{H}}\right]^{2}\right) f_{\mathrm{C}}$; this means that $\left[f_{\text {vir }}\right]$ is smaller than 0.1 for all these groups (see Tables 3-6), except NGC 2403.

\subsection{Mass estimations considering surface effects}

In Sect. 3.3 we proposed the mass estimator $\left\{M_{\mathrm{s}}\right\}_{\beta}$ given by Eq. (73). This mass estimator takes into account surface effects. As commented in that subsection, Eq. (73) has a solution for a range of anisotropy parameters. The range of values allowed for $\beta$ is fixed by two conditions: 1 ) the surfaces terms must be positive or zero (galaxy groups are supposed to be isolated); 2) $\left\{M_{\mathrm{s}}\right\} \geq M_{\mathrm{g}} \approx M_{\mathrm{g}}^{\star}$.
Table 4. Same as Table 3 for the Centaurus A and IC 342 groups.

\begin{tabular}{clrr}
\hline \hline Model & Parameter & Cen A & IC 342 \\
\hline$\left[R_{\mathrm{H}}\right]_{\mathrm{MG}}$ & $\left\{M_{\text {vir }}\right\}$ & 24.31 & 2.86 \\
\hline$[\alpha]_{\mathrm{MG}}$ & $\left\{M_{\text {proj }}\right\}_{\beta=-\infty}$ & 20.73 & 8.78 \\
{$[\alpha]_{\mathrm{MG}}$} & $\left\{M_{\text {proj }}\right\}_{\beta=0}$ & 30.78 & 12.64 \\
{$[\alpha]_{\mathrm{MG}}$} & $\left\{M_{\text {proj }}\right\}_{\beta=1}$ & 60.92 & 24.22 \\
\hline$[\alpha]_{\mathrm{EM}}$ & $\left\{M_{\text {proj }}\right\}_{\beta=-\infty}$ & 38.93 & 16.71 \\
{$[\alpha]_{\mathrm{EM}}$} & $\left\{M_{\text {proj }}\right\}_{\beta=0}$ & 57.80 & 24.05 \\
{$[\alpha]_{\mathrm{EM}}$} & $\left\{M_{\text {proj }}\right\}_{\beta=1}$ & 114.4 & 46.10 \\
\hline & {$\left[f_{\text {vir }}\right]$} & 0.02 & 0.09 \\
& {$\left[f_{\text {proj }}\right]_{\beta=-\infty}$} & 0.03 & 0.14 \\
& {$\left[f_{\text {proj }}\right]_{\beta=0}$} & 0.02 & 0.09 \\
& {$\left[f_{\text {proj }}\right]_{\beta=1}$} & 0.01 & 0.05 \\
\hline
\end{tabular}

Table 5. Same as Table 3 for the probably non-virialized groups NGC 2403, Sculptor Filament, and CVn I Cloud.

\begin{tabular}{clrrr}
\hline \hline Model & Parameter & NGC 2403 & Sculptor & CVn I \\
\hline$\left[R_{\mathrm{H}}\right]_{\mathrm{MG}}$ & $\left\{M_{\text {vir }}\right\}$ & 2.84 & 13.08 & 17.78 \\
{$\left[R_{\mathrm{H}}\right]_{\mathrm{EM}}$} & $\left\{M_{\text {vir }}\right\}$ & 5.32 & 23.22 & 28.22 \\
\hline$[\alpha]_{\mathrm{MG}}$ & $\left\{M_{\text {proj }}\right\}_{\beta=-\infty}$ & 2.45 & 11.93 & 14.28 \\
{$[\alpha]_{\mathrm{MG}}$} & $\left\{M_{\text {proj }}\right\}_{\beta=0}$ & 3.27 & 17.53 & 20.67 \\
{$[\alpha]_{\mathrm{MG}}$} & $\left\{M_{\text {proj }}\right\}_{\beta=1}$ & 5.76 & 34.32 & 39.83 \\
\hline$[\alpha]_{\mathrm{EM}}$ & $\left\{M_{\text {proj }}\right\}_{\beta=-\infty}$ & 4.58 & 21.19 & 22.67 \\
{$[\alpha]_{\mathrm{EM}}$} & $\left\{M_{\text {proj }}\right\}_{\beta=0}$ & 6.13 & 31.13 & 32.80 \\
{$[\alpha]_{\mathrm{EM}}$} & $\left\{M_{\text {proj }}\right\}_{\beta=1}$ & 10.79 & 60.95 & 63.20 \\
\hline & {$\left[f_{\text {vir }}\right]_{1}$} & 0.47 & 0.08 & 0.11 \\
& {$\left[f_{\text {proj }}\right]_{\beta=-\infty}$} & 0.47 & 0.07 & 0.12 \\
& {$\left[f_{\text {proj }}\right]_{\beta=0}$} & 0.32 & 0.04 & 0.08 \\
& {$\left[f_{\text {proj }}\right]_{\beta=1}$} & 0.16 & 0.02 & 0.04 \\
\hline & & & &
\end{tabular}

The results are presented in Tables 6-8 for neighbouring galaxy groups. The tables are composed of panels that correspond to different models. Model parameters $\left[R_{\mathrm{H}}\right]$ and $[\alpha]$ are shown in the note appearing at the bottom of each table. In the first row of panels, we show $[\gamma]=[\alpha]\left[R_{\mathrm{H}}\right] / R_{\mathrm{s}}$; these values are used to determine ranges of $\beta$ (see Sect. 3.3). The minimum and maximum anisotropy parameters allowed, $\beta_{\mathrm{m}}$ and $\beta_{\mathrm{M}}$, are presented in rows 2 and 3. Sample masses for $\beta_{\mathrm{m}}$ and $\beta_{\mathrm{M}}$ appear in rows 4 and 5. Sample masses, when isotropy is assumed, are presented in row 6 (if allowed). Finally, the contributions of cosmological constant terms to $\left\{M_{\mathrm{s}}\right\}_{\beta_{\mathrm{m}}},\left\{M_{\mathrm{s}}\right\}_{\beta_{\mathrm{M}}}$ and $\left\{M_{\mathrm{s}}\right\}_{\beta=0}$ are shown in rows 7-9.

Tables 6 and 7 are devoted to virialized groups, and Table 8, to groups whose virialization is doubtful. Because our study is only applicable to steady groups, the sample masses for nonvirialized groups are questionable; hence, we thought that it was unnecessary to show results for all the models. Thus, in Table 8 , we only present results for the model $\left[R_{\mathrm{H}}\right]_{\mathrm{EM}},[\alpha]_{\mathrm{MG}}$; this is the only model of those considered that allows isotropy for NGC 2403 and CVn I.

We can see from Tables $6-7$ that $[\alpha]_{\text {EM }}$ models show ranges of anisotropy parameters addressed towards values of $\beta$ equal to or smaller than those shown by $[\alpha]_{\mathrm{MG}}$ models (i.e. they show equal or smaller values of $\beta_{\mathrm{m}}$ and $\beta_{\mathrm{M}}$ ). This behaviour has also been observed for the questionable virialization groups.

The models used in the virialized galaxy groups that appear in Tables 6 and 7 show values of $\gamma$ smaller than unity; i.e. $[\alpha]\left[R_{\mathrm{H}}\right]<R_{\mathrm{S}}$ (see Eq. (74)). From these tables, different relations between sample masses for $\beta_{\mathrm{m}}$ and $\beta_{\mathrm{M}}$, projected masses for $\beta_{\mathrm{m}}$ and $\beta_{\mathrm{M}}$, virial masses and galaxy total indicative masses 
Table 6. Sample mass estimator results for the Milky Way, M 31, M 81, M 83, and Maffei groups.

\begin{tabular}{clrrrrr}
\hline \hline Model & Parameter & MW & M31 & M 81 & M 83 & Maffei \\
\hline I & {$[\gamma]$} & 0.30 & 0.15 & 0.32 & 0.52 & 0.71 \\
I & $\beta_{\mathrm{m}}$ & -1.16 & $-\infty$ & -0.81 & 0.02 & - \\
I & $\beta_{\mathrm{M}}$ & 1.00 & 0.98 & 1.00 & 0.96 & - \\
I & $\left\{M_{\mathrm{s}}\right\}_{\beta_{\mathrm{m}}}$ & 8.73 & 5.22 & 9.29 & 9.97 & - \\
I & $\left\{M_{\mathrm{s}}\right\}_{\beta_{\mathrm{M}}}$ & 3.77 & 2.91 & 3.67 & 0.93 & - \\
I & $\left\{M_{\mathrm{s}}\right\}_{\beta=0}$ & 8.06 & 4.62 & 8.66 & - & - \\
I & {$\left[f_{\mathrm{s}}\right]_{\beta_{\mathrm{m}}}$} & 0.01 & 0.01 & 0.00 & 0.01 & - \\
I & {$\left[f_{\mathrm{s}}\right]_{\beta_{\mathrm{M}}}$} & 0.01 & 0.02 & 0.01 & 0.13 & - \\
I & {$\left[f_{\mathrm{s}}\right]_{\beta=0}$} & 0.01 & 0.01 & 0.00 & - & - \\
\hline II & {$[\gamma]$} & 0.15 & 0.08 & 0.17 & 0.26 & 0.40 \\
II & $\beta_{\mathrm{m}}$ & $-\infty$ & $-\infty$ & $-\infty$ & $-\infty$ & 0.95 \\
II & $\beta_{\mathrm{M}}$ & 1.00 & 0.94 & 1.00 & 0.94 & 1.00 \\
II & $\left\{M_{\mathrm{s}}\right\}_{\beta_{\mathrm{m}}}$ & 7.83 & 4.81 & 8.40 & 8.86 & 5.58 \\
II & $\left\{M_{\mathrm{s}}\right\}_{\beta_{\mathrm{M}}}$ & 3.11 & 2.91 & 2.98 & 0.93 & 5.33 \\
II & $\left\{M_{\mathrm{s}}\right\}_{\beta=0}$ & 6.65 & 4.25 & 7.05 & 6.56 & - \\
II & {$\left[f_{\mathrm{s}}\right]_{\beta_{\mathrm{m}}}$} & 0.01 & 0.01 & 0.00 & 0.01 & 0.01 \\
II & {$\left[f_{\mathrm{s}}\right]_{\beta_{\mathrm{M}}}$} & 0.01 & 0.01 & 0.01 & 0.08 & 0.01 \\
II & {$\left[f_{\mathrm{s}}\right]_{\beta=0}$} & 0.01 & 0.01 & 0.00 & 0.01 & - \\
\hline
\end{tabular}

Notes. Model I: $\left[R_{\mathrm{H}}\right]_{\mathrm{EM}},[\alpha]_{\mathrm{MG}}$. Model II: $\left[R_{\mathrm{H}}\right]_{\mathrm{EM}},[\alpha]_{\mathrm{EM}}$. Sample masses are given in units of $10^{11} M_{\odot}$.

Table 7. Sample mass estimator results for the Centaurus A and IC 342.

\begin{tabular}{clrr}
\hline \hline Model & Parameter & Cen A & IC 342 \\
\hline I & {$[\gamma]$} & 0.46 & 0.17 \\
I & $\beta_{\mathrm{m}}$ & -2.40 & $-\infty$ \\
I & $\beta_{\mathrm{M}}$ & 0.80 & -0.43 \\
I & $\left\{M_{\mathrm{s}}\right\}_{\beta_{\mathrm{m}}}$ & 24.31 & 1.68 \\
I & $\left\{M_{\mathrm{s}}\right\}_{\beta_{\mathrm{M}}}$ & 6.09 & 1.09 \\
I & $\left\{M_{\mathrm{s}}\right\}_{\beta=0}$ & 18.79 & - \\
I & {$\left[f_{\mathrm{s}}\right]_{\beta_{\mathrm{m}}}$} & 0.01 & 0.05 \\
I & {$\left[f_{\mathrm{s}}\right]_{\beta_{\mathrm{M}}}$} & 0.04 & 0.08 \\
I & {$\left[f_{\mathrm{s}}\right]_{\beta=0}$} & 0.01 & - \\
\hline II & {$[\gamma]$} & 0.25 & 0.09 \\
II & $\beta_{\mathrm{m}}$ & $-\infty$ & $-\infty$ \\
II & $\beta_{\mathrm{M}}$ & 0.73 & -0.81 \\
II & $\left\{M_{\mathrm{s}}\right\}_{\beta_{\mathrm{m}}}$ & 19.60 & 1.53 \\
II & $\left\{M_{\mathrm{s}}\right\}_{\beta_{\mathrm{M}}}$ & 6.09 & 1.09 \\
II & $\left\{M_{\mathrm{s}}\right\}_{\beta=0}$ & 13.43 & - \\
II & {$\left[f_{\mathrm{s}}\right]_{\beta_{\mathrm{m}}}$} & 0.01 & 0.05 \\
II & {$\left[f_{\mathrm{s}}\right]_{\beta_{\mathrm{M}}}$} & 0.03 & 0.07 \\
II & {$\left[f_{\mathrm{s}}\right]_{\beta=0}$} & 0.01 & - \\
\hline
\end{tabular}

Notes. Model I: $\left[R_{\mathrm{H}}\right]_{\mathrm{MG}},[\alpha]_{\mathrm{MG}}$. Model II: $\left[R_{\mathrm{H}}\right]_{\mathrm{MG}},[\alpha]_{\mathrm{EM}}$. Sample masses are given in units of $10^{11} M_{\odot}$.

can be appreciated. Thus, for $\beta_{\mathrm{m}} \neq-\infty, \beta_{\mathrm{M}}=1$,

$M_{\mathrm{g}}^{\star}<\left\{M_{\mathrm{s}}\right\}_{\beta_{\mathrm{M}}}<\left\{M_{\mathrm{s}}\right\}_{\beta_{\mathrm{m}}}=\left\{M_{\mathrm{vir}}\right\}=\left\{M_{\text {proj }}\right\}_{\beta_{\mathrm{m}}}<\left\{M_{\text {proj }}\right\}_{\beta_{\mathrm{M}}} ;$

for $\beta_{\mathrm{m}} \neq-\infty, \beta_{\mathrm{M}}<1$,

$M_{\mathrm{g}}^{\star}=\left\{M_{\mathrm{s}}\right\}_{\beta_{\mathrm{M}}}<\left\{M_{\mathrm{s}}\right\}_{\beta_{\mathrm{m}}}=\left\{M_{\mathrm{vir}}\right\}=\left\{M_{\text {proj }}\right\}_{\beta_{\mathrm{m}}}<\left\{M_{\text {proj }}\right\}_{\beta_{\mathrm{M}}} ;$

for $\beta_{\mathrm{m}}=-\infty, \beta_{\mathrm{M}}<1$,

$M_{\mathrm{g}}^{\star}=\left\{M_{\mathrm{s}}\right\}_{\beta_{\mathrm{M}}}<\left\{M_{\mathrm{s}}\right\}_{\beta_{\mathrm{m}}}<\left\{M_{\mathrm{vir}}\right\}<\left\{M_{\text {proj }}\right\}_{\beta_{\mathrm{m}}}<\left\{M_{\text {proj }}\right\}_{\beta_{\mathrm{M}}} ;$

and finally, for $\beta_{\mathrm{m}}=-\infty, \beta_{\mathrm{M}}=1$,

$M_{\mathrm{g}}^{\star}<\left\{M_{\mathrm{s}}\right\}_{\beta_{\mathrm{M}}}<\left\{M_{\mathrm{s}}\right\}_{\beta_{\mathrm{m}}}<\left\{M_{\mathrm{vir}}\right\}<\left\{M_{\text {proj }}\right\}_{\beta_{\mathrm{m}}}<\left\{M_{\text {proj }}\right\}_{\beta_{\mathrm{M}}}$.
Table 8. Sample mass estimator results for the NGC 2403 group, Sculptor Filament, and Canes Vanatici I Cloud.

\begin{tabular}{lrrr}
\hline \hline Parameter & NGC 2403 & Sculptor & CVn I \\
\hline$[\gamma]$ & 1.96 & 1.31 & 1.54 \\
$\beta_{\mathrm{m}}$ & -1.05 & 0.12 & -0.63 \\
$\beta_{\mathrm{M}}$ & 0.95 & 0.67 & 0.72 \\
$\left\{M_{\mathrm{s}}\right\}_{\beta_{\mathrm{m}}}$ & 0.41 & 1.32 & 0.72 \\
$\left\{M_{\mathrm{s}}\right\}_{\beta_{\mathrm{M}}}$ & 5.32 & 23.22 & 28.22 \\
$\left\{M_{\mathrm{s}}\right\}_{\beta=0}$ & 1.16 & - & 6.60 \\
{$\left[f_{\mathrm{s}}\right]_{\beta_{\mathrm{m}}}$} & -0.29 & -0.62 & -0.46 \\
{$\left[f_{\mathrm{s}}\right]_{\beta_{\mathrm{M}}}$} & -0.03 & -0.09 & -0.02 \\
{$\left[f_{\mathrm{s}}\right]_{\beta=0}$} & -0.13 & - & -0.09 \\
\hline
\end{tabular}

Notes. Model: $\left[R_{\mathrm{H}}\right]_{\mathrm{EM}},[\alpha]_{\mathrm{MG}}$. Sample masses are given in units of $10^{11} M_{\odot}$

Relations given by Eqs. (105)-(108) are a consequence of Eqs. (71) and (72).

Some models proposed for dealing with galaxy groups with questionable virialization also show $\gamma<1$; hence, relations (105)-(108) are also fulfilled. However, other models present $\gamma>1$. For these last models, the only relation that can be deduced from the results that appear in Table 8 is

$M_{\mathrm{g}}^{\star}=\left\{M_{\mathrm{s}}\right\}_{\beta_{\mathrm{m}}}<\left\{M_{\text {proj }}\right\}_{\beta_{\mathrm{m}}}<\left\{M_{\mathrm{s}}\right\}_{\beta_{\mathrm{M}}}=\left\{M_{\text {proj }}\right\}_{\beta_{\mathrm{M}}}=\left\{M_{\mathrm{vir}}\right\}$,

for $\beta_{\mathrm{m}} \neq-\infty, \beta_{\mathrm{M}}<1$.

We note from Table 6 that Maffei has no solution for model $[\alpha]_{\mathrm{MG}}$; and, for model $[\alpha]_{\mathrm{EM}}$, the permitted average Keplerian orbits are close to radial ones. In the same table, we also see that the M 83 group treated by model $[\alpha]_{\mathrm{MG}}$ shows permitted anisotropy parameters where the radial velocity dispersion dominates.

In Table 7, it is noticeable that tangential velocity dispersion dominates anisotropy parameters for IC 342.

For the virialized galaxy groups shown in Tables 6 and 7 , the contributions of cosmological constant terms to the sample masses are positive. They are smaller than 0.02 for the Milky Way, M 31, M 81, and Maffei. For M 83, Centaurus A, and IC 342 they range from 0.01 up to 0.13 . These results indicate that the use of mass estimators which do not include $\Lambda$-terms is not a bad approximation, bearing in mind that these mass estimators already include some effect of $\Lambda$ because they use observational data.

We have seen that the Milky Way, M 31, M 81, IC 342, and Maffei groups fulfill $\left[f_{\text {vir }}\right]<\left[f_{\text {proj }}\right]_{\beta_{\mathrm{M}}}<\left[f_{\text {proj }}\right]_{\beta_{\mathrm{m}}}$ and $\left[f_{\mathrm{s}}\right]_{\beta_{\mathrm{m}}}<$ $\left[f_{\mathrm{s}}\right]_{\beta_{\mathrm{M}}}<\left[f_{\text {vir }}\right]$; while M 83 and Centaurus A show $\left[f_{\text {proj }}\right]_{\beta_{\mathrm{M}}}<$ $\left[f_{\text {vir }}\right]<\left[f_{\text {proj }}\right]_{\beta_{\mathrm{m}}}$ and $<\left[f_{\mathrm{s}}\right]_{\beta_{\mathrm{m}}}<\left[f_{\text {vir }}\right]<\left[f_{\mathrm{s}}\right]_{\beta_{\mathrm{M}}}$.

Some models used in groups with questionable virialization lead to negative values of $\left[f_{\mathrm{s}}\right]$. This is due to the denominator signus of Eq. (78). We have seen that models with $\gamma>1$ present $\left[f_{\mathrm{s}}\right]<0 ; 0.7<\gamma<1$ models show both positive and negative values; while for $\gamma<0.7$, they are positive. These groups present $\left|\left[f_{\mathrm{s}}\right]\right| \mathrm{s}$ which are much greater than those shown by the majority of the virialized groups because the denominator of Eq. (78) is closer to zero.

NGC 2403, Sculptor, and CVn I show different relations between the cosmological constant term contributions to the virial and projected masses for $\beta_{\mathrm{m}}$ and $\beta_{\mathrm{M}}$ than those shown by the virialized groups. Thus, $\left[f_{\text {proj }}\right]_{\beta_{\mathrm{M}}}<\left[f_{\text {proj }}\right]_{\beta_{\mathrm{m}}}<\left[f_{\text {vir }}\right]$ is fulfilled by these groups. The relations between $\left[f_{\mathrm{vir}}\right],\left[f_{\mathrm{s}}\right]_{\beta_{\mathrm{m}}}$, and $\left[f_{\mathrm{s}}\right]_{\beta_{\mathrm{M}}}$ are also different. 
Table 9. Sample mass in units of $10^{11} M_{\odot}$ proposed for some virialized galaxy groups from models assuming $[\alpha]_{\mathrm{MG}}$.

\begin{tabular}{cccc}
\hline \hline Group & Model & $\beta$ & $\left\{M_{\mathrm{s}}\right\}$ \\
\hline MW & {$\left[R_{\mathrm{H}}\right]_{\mathrm{EM}}$} & 1.00 & 3.8 \\
M 31 & {$\left[R_{\mathrm{H}}\right]_{\mathrm{EM}}$} & 0.00 & 4.6 \\
M 81 & {$\left[R_{\mathrm{H}}\right]_{\mathrm{EM}}$} & 0.00 & 8.7 \\
M 83 & {$\left[R_{\mathrm{H}}\right]_{\mathrm{EM}}$} & 0.02 & 10.0 \\
Cen A & {$\left[R_{\mathrm{H}}\right]_{\mathrm{MG}}$} & 0.00 & 18.8 \\
IC 342 & {$\left[R_{\mathrm{H}}\right]_{\mathrm{MG}}$} & $-0.43--\infty$ & $1.1-1.7$ \\
\hline
\end{tabular}

Table 10. Same as Table 9, but from models assuming $[\alpha]_{\mathrm{EM}}$.

\begin{tabular}{cccc}
\hline \hline Group & Model & $\beta$ & $\left\{M_{\mathrm{s}}\right\}$ \\
\hline M 81 & {$\left[R_{\mathrm{H}}\right]_{\mathrm{EM}}$} & 0.00 & 7.0 \\
M 83 & {$\left[R_{\mathrm{H}}\right]_{\mathrm{EM}}$} & 0.00 & 6.6 \\
Maffei & {$\left[R_{\mathrm{H}}\right]_{\mathrm{EM}}$} & $1.00-0.95$ & $5.3-5.6$ \\
Cen A & {$\left[R_{\mathrm{H}}\right]_{\mathrm{MG}}$} & 0.00 & 13.4 \\
IC 342 & {$\left[R_{\mathrm{H}}\right]_{\mathrm{MG}}$} & $-0.81--\infty$ & $1.1-1.5$ \\
\hline
\end{tabular}

\subsection{Sample masses proposed for neighbouring virialized galaxy groups}

Tables 9 and 10 show the sample masses that we propose for the set of virialized groups addressed in this work. The results presented in Table 9 come from models assuming $[\alpha]_{\mathrm{MG}}$. Those shown in Table 10 correspond to models $[\alpha]_{\text {EM }}$. Sample masses are presented in the fourth columns of Tables 9 and 10. The second column indicates the model $\left[R_{\mathrm{H}}\right]$ assumed to derive them and the third column presents anisotropy parameters corresponding to those masses. The proposed sample masses can be compared with the virial and projected estimations presented in Tables 3 and 4 (use Eq. (67) to calculate $\left\{M_{\text {proj }}\right\}_{\beta_{\mathrm{m}}}$ and $\left\{M_{\text {proj }}\right\}_{\beta_{\mathrm{M}}}$ ).

The masses appearing in Tables 9 and 10 have been selected from the results presented in Tables 6 and 7, as described in the below paragraphs. In the case of absence of information about the group anisotropy parameter, values assuming isotropy are taken. This seems to be the best choice (see, for example, Heisler et al. 1985). If isotropy is not allowed, the whole range of sample mass values are considered. NGC 2403, Sculptor, and $\mathrm{CVn}$ I are not addressed in this subsection because these groups could be non-virialized.

We note that all companions to the Milky Way, except NGC 6822, are located in an elongated volume called the polar Magellanic Stream; thus, if the orbits are strongly elongated, we see almost the full vectors of their velocities. Hence, the anisotropy parameter of the group could be close to unity. When dealing with this group, Karachentsev (2005) take into account the expected mass ratio 4:5 for the Milky Way and M 31 groups. He accepts his virial and projected mass estimation of the M31 group and rejects his results for the Milky Way group. Looking at Table 6 , we can compare $\left\{M_{\mathrm{s}}\right\}_{\beta=1}$ for the Milky Way with $\left\{M_{\mathrm{s}}\right\}_{\beta=0}$ for M 31 . For models $\left[R_{\mathrm{H}}\right]_{\mathrm{EM}},[\alpha]_{\mathrm{MG}}$, the mass ratio is 4.1:5, while for models $\left[R_{\mathrm{H}}\right]_{\mathrm{EM}},[\alpha]_{\mathrm{EM}}$ it is 3.7:5. As can be seen, both mass ratios are in agreement with that expected. In this work, we adopt results leading to a mass ratio 4.1:5. Thus, the surface term effects on the Milky Way group make that its sample mass estimation represents about $44 \%$ of the virial mass estimation and $19 \%$ of the projected mass $(\beta=1)$. With respect to M 31 , it is about $83 \%$ of the virial mass and $42 \%$ of the projected mass $(\beta=0)$.

For the M 81 and M 83 groups, we take the sample mass estimations for isotropy shown in Table 6. We note that the model $\left[R_{\mathrm{H}}\right]_{\mathrm{EM}},[\alpha]_{\mathrm{MG}}$ for M 83 leads to $\beta_{\mathrm{m}}=0.02$; we take the sample mass corresponding to this anisotropy parameter as that of isotropy. For M 81, when model $[\alpha]_{\mathrm{MG}}$ is considered, the sample mass estimation is about $94 \%$ of the virial mass and $82 \%$ of the projected mass $(\beta=0)$; when model $[\alpha]_{\mathrm{EM}}$ is assumed, it represents $75 \%$ and $35 \%$. For M 83 , the sample mass, virial mass, and projected mass $(\beta=0)$ estimations are similar when using model $[\alpha]_{\mathrm{MG}}$; for model $[\alpha]_{\mathrm{EM}}$, the sample mass is about $66 \%$ of the virial mass and $34 \%$ of the projected mass.

From our study, we have seen that the Maffei group has no solution for $\beta=0$. For this group, we have presented the whole range of permitted sample masses in Table 10. For $\beta_{\mathrm{m}}=0.95$, the sample mass, virial mass, and project mass coincide; and, for $\beta_{\mathrm{M}}=1$, the sample mass represents about $95 \%$ and $87 \%$ of the virial and projected mass estimations, respectively.

For Centaurus A, $\left\{M_{\mathrm{s}}\right\}_{\beta=0}$ estimations appearing in Table 7 are also shown in Tables 9 and 10 . When model $[\alpha]_{\mathrm{MG}}$ is assumed, the sample mass represents about $77 \%$ of the virial mass and $61 \%$ of the projected mass $(\beta=0)$; and for model $[\alpha]_{\mathrm{EM}}$, it represents $55 \%$ and $23 \%$.

Table 7 indicates that isotropy is not allowed in the IC 342 group. Hence, we consider the whole range of sample mass. For model $[\alpha]_{\mathrm{MG}}$, the sample mass estimation for $\beta_{\mathrm{m}}=-\infty$ is about $59 \%$ of the virial mass and $19 \%$ of the projected mass; and for $\beta_{\mathrm{M}}=-0.43$, it represents $38 \%$ and $9 \%$. In the case of assuming model $[\alpha]_{\mathrm{EM}}$, the sample mass for $\beta_{\mathrm{m}}=-\infty$ is $53 \%$ of the virial mass and $9 \%$ of the projected mass; and for $\beta_{\mathrm{M}}=-0.81$, it represents $38 \%$ of the virial mass and $5 \%$ of the projected mass.

\section{Comparison with neighbouring galaxy complex masses derived from turn-around radius data}

\subsection{Virialized masses from turn-around radii}

For the sake of simplicity, the collapse of cold dark matter $(\mathrm{CDM})$ can be treated with spherical infall models (see, for example, Peebles 1980, Sect. 19). These models study the nonlinear collapse of a CDM spherical shell. For this purpose, its motion equation is integrated from redshift $z_{\mathrm{I}}=1000$, in a background of matter and cosmological constant, and assuming that no other mass shell crosses it.

The mass enclosed by a shell $i, M_{i}$, together with the profile of the collapsing structure at $z_{\mathrm{I}}$, fix the shell radius and its proper velocity at that redshift. This proper velocity should decrease until taking a value equal to zero at some $z_{i}^{\mathrm{TA}}$. At this redshift, the shell radius is maximum and is known as the turn-around radius, $r_{i}^{\mathrm{TA}}$. From $z_{i}^{\mathrm{TA}}$, the radius of the shell decreases. The collapse ends when a virialized structure with a virial radius, $r_{i}^{\mathrm{VIR}}$, is formed at $z_{i}^{\mathrm{VIR}}$. We note that this does not occur for all shells. The repulsive effect of the cosmological constant makes massive shells reach zero proper acceleration and keep expanding without reaching a turn-around radius.

Membrado \& Pacheco (2014) studied the influence of the cosmological constant on the non-linear collapse of CDM clusters. For an average cluster, they obtained as a function of the redshift, relations among the radii and masses of the shell that reaches its turn-around point and the shell that virializes. At $z=0$, assuming $\Omega_{\Lambda}=0.73$ and $H_{0}=71 \mathrm{~km} \mathrm{~s}^{-1} \mathrm{Mpc}^{-1}$, they find

$\left(\frac{M^{\mathrm{TA}}}{10^{15} M_{\odot}}\right)=2.10\left(\frac{r^{\mathrm{TA}}}{10 \mathrm{Mpc}}\right)^{3}$,

$M^{\mathrm{TA}}=1.95 M^{\mathrm{VIR}}$. 
Table 11. Neighbouring galaxy complex masses.

\begin{tabular}{llccccccc}
\hline \hline Parameter & Units & $\begin{array}{c}\text { MW } \\
\text { M 31 }\end{array}$ & $\begin{array}{c}\text { M 81 } \\
\text { NGC 2403 }\end{array}$ & $\begin{array}{c}\text { Cen A } \\
\text { M 83 }\end{array}$ & $\begin{array}{c}\text { IC 342 } \\
\text { Maffei }\end{array}$ & $\begin{array}{c}\text { Average } \\
\text { (virialized complexes) }\end{array}$ & Sculptor & CVn I \\
\hline$r^{\mathrm{TA}}$ & Mpc & $0.94 \pm 0.10$ & $1.05 \pm 0.07$ & $1.26 \pm 0.15$ & $0.90 \pm 0.10$ & $1.04 \pm 0.11$ & $0.70 \pm 0.10$ & $0.63 \pm 0.10$ \\
$M^{\mathrm{TA}}$ & $10^{11} M_{\odot}$ & $17.4 \pm 5.6$ & $24.3 \pm 4.9$ & $42.0 \pm 15.0$ & $15.3 \pm 5.1$ & $24.8 \pm 7.6$ & $7.2 \pm 3.1$ & $5.3 \pm 2.5$ \\
$M^{\mathrm{VIR}}$ & $10^{11} M_{\odot}$ & $8.9 \pm 2.8$ & $12.5 \pm 2.5$ & $21.5 \pm 7.7$ & $7.9 \pm 2.6$ & $12.7 \pm 3.9$ & \\
\hline $\max \left(\sum\left\{M_{\mathrm{s}}\right\}\right)$ & $10^{11} M_{\odot}$ & 8.4 & 9.1 & 28.8 & 7.3 & 13.4 & \\
$\min \left(\sum\left\{M_{\mathrm{s}}\right\}\right)$ & $10^{11} M_{\odot}$ & 8.4 & 8.2 & 20.0 & 6.4 & 10.8 & \\
\hline
\end{tabular}

Equation (110) is comparable with that derived from the work by Rines et al. (2003) (based on data from the Cluster and Infall Region Nearby Survey). Equation (111) is in agreement with the results reported by Rines \& Diaferio (2006) (from the Fourth Data Release of the Sloan Digital Sky Survey).

In Membrado \& Pacheco (2014), the virial radius for a mass $M_{i}$ was estimated by imposing the conservation of the energy between $z_{i}^{\mathrm{TA}}$ and $z_{i}^{\mathrm{VIR}}$. At the turn-around point, the kinetic energy of the shell $i$ is zero; there, a mass sphere of constant density was assumed. At the virial redshift, the kinetic energy was calculated by imposing the virial theorem under steady conditions and including the surface term.

When the virial theorem was applied, a singular isothermal sphere of mass was assumed. Hence, the virial relation was that given by Eq. (10), i.e.

$M_{i}=\frac{2}{G} \sigma_{\mathrm{los}, i}^{2} r_{i}^{\mathrm{VIR}}\left[1+\frac{4 \pi G \rho_{\Lambda}}{9 c^{2}} \frac{\left(r_{i}^{\mathrm{VIR}}\right)^{2}}{\sigma_{\mathrm{los}, i}^{2}}\right]$.

As can be seen, the contribution of the cosmological constant term is negligible when Eq. (112) is applied to galaxy clusters (take, for example, $r_{i}^{\mathrm{VIR}}=5 \mathrm{Mpc}$ and $\sigma_{\mathrm{los}, i}=1000 \mathrm{~km} \mathrm{~s}^{-1}$ ). In the previous section, we saw that the term is also negligible for the virialized neighbouring groups of galaxies.

Although the contribution of the cosmological constant term to the virial mass is not appreciable, the evolution of a mass cluster in a cosmological background of matter and cosmological constant causes the virial mass to differ from that derived from a $\Lambda=0$ model. This can be seen when comparing Eqs. (110) and (111) with the results for the $\Lambda=0$ model:

$\left(\frac{\left[M^{\mathrm{TA}}\right]_{\Lambda=0}}{10^{15} M_{\odot}}\right)=3.27\left(\frac{\left[r^{\mathrm{TA}}\right]_{\Lambda=0}}{10 \mathrm{Mpc}}\right)^{3}$,

$\left[M^{\mathrm{TA}}\right]_{\Lambda=0}=5.26\left[M^{\mathrm{VIR}}\right]_{\Lambda=0}$.

Karachentsev et al. (2002a,b,c, 2003a,b,c,d) determined turnaround radii at $z \approx 0$ for neighbouring complexes of galaxies that include the groups addressed in this work. From these data, Karachentsev (2005) estimated masses for these complexes using the Lynden-Bell (1981) and Sandage (1986) relation (depending on the age of the Universe):

$\left(\frac{\left[M^{\mathrm{TA}}\right]_{\Lambda=0}}{10^{15} M_{\odot}}\right)=1.46\left(\frac{\left[r^{\mathrm{TA}}\right]_{\Lambda=0}}{10 \mathrm{Mpc}}\right)^{3}$.

As can be seen, Eqs. (115) and (113) differ appreciably. We should mention that Karachentsev \& Nasonova (2010) derived another analytical expression, which depends on $H_{0}$ and $\Omega_{\Lambda}$. From their equation, and assuming $H_{0}=71 \mathrm{~km} \mathrm{~s}^{-1} \mathrm{Mpc}^{-1}$, it can be found that for $\Omega_{\Lambda}=0.73$ and for $\Omega_{\Lambda}=0$,

$\left(\frac{M^{\mathrm{TA}}}{10^{15} M_{\odot}}\right)=2.16\left(\frac{r^{\mathrm{TA}}}{10 \mathrm{Mpc}}\right)^{3}$,

$\left(\frac{\left[M^{\mathrm{TA}}\right]_{\Lambda=0}}{10^{15} M_{\odot}}\right)=3.24\left(\frac{\left[r^{\mathrm{TA}}\right]_{\Lambda=0}}{10 \mathrm{Mpc}}\right)^{3}$.
Thus, Eqs. (116) and (117) are in agreement with Eqs. (110) and (113).

Equations (110) and (111) are relations derived for galaxy clusters at present. In the following calculations, we assumed that these equations are also fulfilled by galaxy groups at present. Thus,

$$
M^{\mathrm{TA}}=2.10 \times 10^{12} M_{\odot}\left(\frac{r^{\mathrm{TA}}}{1 \mathrm{Mpc}}\right)^{3},
$$

$M^{\mathrm{VIR}}=5.13 \times 10^{-1} M^{\mathrm{TA}}$.

Rows 1-3 of Table 11 (upper panel) show the turn-around radii, $r^{\mathrm{TA}}$, turn-around masses, $M^{\mathrm{TA}}$, and virialized masses, $M^{\mathrm{VIR}}$, of several galaxy complexes. Again, for a galaxy complex, $r^{\mathrm{TA}}$ and $M^{\mathrm{TA}}$ are the radius of the shell reaching its turnaround point at present, and the mass contained in the sphere of such a radius; and, $M^{\mathrm{VIR}}$ is the mass contained in the sphere covered by the shell which is virializing at $z=0$. Columns 3-6 are devoted to the virialized complexes Milky Way - M31, M 81 - NGC 2403, Centaurus A - M 83, and IC 342 - Maffei. Columns 8 and 9 show quantities for the expanding complexes Sculptor Filament and Canes Venatici I Cloud (virial masses for these systems have not been calculated). Column 7 presents quantities averaged in the number of virialized complexes. The turn-around radii are taken from Karachentsev (2005). Using these quantities in Eqs. (118), (119), the turn-around masses and virialized masses are derived. Our $M^{\mathrm{TA}}$ and $M^{\mathrm{VIR}}$ results for virialized complexes differ from the masses calculated by Karachentsev (2005). His results obtained by using Eq. (115) are between our turn-around and virialized mass estimations.

For the average galaxy cluster, Membrado \& Pacheco (2014) obtained $M^{\mathrm{VIR}}=1.03 \times 10^{14} M_{\odot}\left(r^{\mathrm{VIR}} / 1 \mathrm{Mpc}\right)^{3}$ at $z=0$. Using this relation and the value of the critical energy density at present,

$\frac{\rho_{0}}{c^{2}}=\frac{3 H_{0}^{2}}{8 \pi G}=1.4 \times 10^{11}\left(\frac{H_{0}}{71 \mathrm{~km} \mathrm{~s}^{-1} \mathrm{Mpc}^{-1}}\right)^{2} M_{\odot} \mathrm{Mpc}^{-3}$,

the virialized mean mass density of galaxy complexes, $\rho^{\mathrm{VIR}}$, is

$\rho^{\mathrm{VIR}}=\frac{3 M^{\mathrm{VIR}}}{4 \pi\left[r^{\mathrm{VIR}}\right]^{3}}=1.8 \times 10^{2} \frac{\rho_{0}}{c^{2}}\left(\frac{H_{0}}{71 \mathrm{~km} \mathrm{~s}^{-1} \mathrm{Mpc}^{-1}}\right)^{-2}$.

From works by Gunn \& Gott (1972) and Crone et al. (1994), it is well known that, on average, practically all the virialized cluster mass is contained inside the radius where mean mass density is greater than $200 \rho_{0} / c^{2}$ (outside this radius, velocities become dominated by infall). As can be seen, Eq. (121) is in the order of this result (taking into account that a singular isothermal sphere of mass was assumed for the virialized structure). 
With respect to the turn-around mass density, $\rho^{\mathrm{TA}}$, from Eqs. (118) and (120),

$\rho^{\mathrm{TA}}=\frac{3 M^{\mathrm{TA}}}{4 \pi\left[r^{\mathrm{TA}}\right]^{3}}=3.6 \frac{\rho_{0}}{c^{2}}\left(\frac{H_{0}}{71 \mathrm{~km} \mathrm{~s}^{-1} \mathrm{Mpc}^{-1}}\right)^{-2}$.

As seen in Table 11, the total mass contained in virialized complexes up to their turn-around-radii is $\left[M^{\mathrm{TA}}\right]_{\mathrm{vir}} \approx 99.0 \times$ $10^{11} M_{\odot}$. This value is about 1.44 times greater than that given by Karachentsev (2005); this is due to differences between Eqs. (115) and (118). Taking into account that the integrated luminosity of these objects is $\left[L_{\mathrm{B}}\right]_{\mathrm{vir}}=3.0 \times 10^{11} L_{\odot}$ (see Karachentsev 2005), the ratio of total mass and total luminosity of the virialized complexes is $\left[M^{\mathrm{TA}}\right]_{\mathrm{vir}} /\left[L_{\mathrm{B}}\right]_{\mathrm{vir}}=33 M_{\odot} / L_{\odot}$.

All the complexes shown in Table 11 are inside a sphere of radius $5 \mathrm{Mpc}\left(V_{5 \mathrm{Mpc}}=523.6 \mathrm{Mpc}^{3}\right)$. In this sphere, the virialized complexes contribute 121 galaxies, and Sculptor and CVn I bring 15; besides, there are 47 galaxies in smaller multiple systems and 50 field galaxies (see Karachentsev 2005). The inclusion of the two possibly unvirialized expanding complexes (Sculptor Filament and CVn I Cloud) in the calculation of the mass-to-luminosity ratio reduces its value. Hence, we could take $33 M_{\odot} / L_{\odot}$ as an upper limit for the mass-to-luminosity ratio in the sphere of $5 \mathrm{Mpc}$.

The integrated luminosity of all the galaxies in the sphere of $5 \mathrm{Mpc}$ is $\left[L_{\mathrm{B}}\right]_{5 \mathrm{Mpc}}=4.6 \times 10^{11} L_{\odot}$ (see Karachentsev et al. 2004). So, using $\left[M^{\mathrm{TA}}\right]_{\mathrm{vir}} /\left[L_{\mathrm{B}}\right]_{\mathrm{vir}}$, the mass contained in the sphere should be smaller than $1.5 \times 10^{13} M_{\odot}$; this quantity leads to a mass density, $\left[\rho_{\mathrm{m}}\right]_{5 \mathrm{Mpc}}<2.9 \times 10^{10} M_{\odot} \mathrm{Mpc}^{-3}$. This value is smaller than the mass density of matter background in the present Universe, $\rho_{\mathrm{m} 0}=\Omega_{\mathrm{m} 0} \rho_{0} / c^{2} \approx 3.8 \times 10^{10} M_{\odot} \mathrm{Mpc}^{-3}$ $\left(\Omega_{\mathrm{m} 0}=0.27\right.$, from Spergel et al. (2003), is assumed). Hence, it should be necessary to assume the existence of another component of dark matter. Karachentsev (2005) was the first author to arrive at this conclusion.

If mass density were proportional to luminosity density on different scales, a mass-to-luminosity ratio could be estimated from the energy density of matter background in the Universe at $z=0$ and the mean luminosity density. The mean luminosity density, $\bar{\rho}_{\mathrm{L}}$, can be estimated from the Sloan Digital Sky Survey (SDSS; Blanton et al. 2003) and the Millennium Galaxy Catalogue (MGC; Liske et al. 2003). When this is corrected by internal extinction in galaxies (see Karachentsev 2005), $\bar{\rho}_{\mathrm{L}} \approx$ $2.0 \times 10^{8} L_{\odot} \mathrm{Mpc}^{-3}$. So, using Eq. (120) and $\Omega_{\mathrm{m} 0}=0.27$, the mean mass-to-luminosity ratio would be

$\left(\frac{M}{L}\right)_{0}=\frac{\rho_{0} \Omega_{\mathrm{m} 0}}{c^{2} \bar{\rho}_{\mathrm{L}}} \approx 1.9 \times 10^{2} M_{\odot} L_{\odot}^{-1}$.

When Eq. (123) is applied to the sphere of $5 \mathrm{Mpc}\left(4.6 \times 10^{11} L_{\odot}\right)$, the mass obtained is $8.7 \times 10^{13} M_{\odot}$; this value leads to a mass density of $1.7 \times 10^{11} M_{\odot} \mathrm{Mpc}^{-3}$. This value is about $1.2\left(\rho_{0} / c^{2}\right)$; i.e. it is 4.5 times greater than the mass density of matter background.

All groups addressed in this work are located in a layer of $\pm 1 / 3 \mathrm{Mpc}$ around the local "pancake", which occupies a volume $V_{\text {layer }}=52.4 \mathrm{Mpc}^{3}$. From the turn-around radii given in Table 11, the four virialized complexes and the two expanding groups would have a total volume of $V_{\mathrm{TA}}=22.2 \mathrm{Mpc}^{3}$. An estimation of the volumen of the layer that is occupied by them can be obtained assuming disks of $2 / 3 \mathrm{Mpc}$ and radii equal to their turn-around radii. Thus, $V_{\mathrm{TA}}$ together with the volumen of the layer, which is not occupied by groups, would be about $V=63.6 \mathrm{Mpc}^{3}$. Assuming that the mass constrained in $V$ is in the order of the sum of the turn-around masses of the four complexes and the two expanding groups, i.e. about $1.12 \times 10^{13} M_{\odot}$,
Table 12. Properties of the M 81 - NGC 2403 complex.

\begin{tabular}{llc}
\hline \hline Parameter & Units & Value \\
\hline$N$ & - & 29 \\
$M_{\mathrm{g}}^{\star}$ & $10^{11} M_{\odot}$ & 2.21 \\
{$\left[m_{\mathrm{g}}^{\star}\right]_{1}$} & $10^{11} M_{\odot}$ & 1.66 \\
{$\left[\sigma_{\text {los }}^{2}\right]^{1 / 2}$} & $\mathrm{~km} \mathrm{~s}^{-1}$ & 90.7 \\
$R_{\mathrm{s}}$ & $\mathrm{Mpc}$ & 0.883 \\
{$[R]$} & $\mathrm{Mpc}$ & 0.266 \\
{$\left[R_{\perp} \sigma_{\text {los }}^{2}\right]$} & $\mathrm{Mpc} \mathrm{km}{ }^{2} \mathrm{~s}^{-2}$ & 1621 \\
{$\left[R_{\mathrm{H}}\right]_{\mathrm{PM}}$} & $\mathrm{Mpc}$ & 0.101 \\
{$\left[R_{\mathrm{H}}\right]_{\mathrm{MG}}$} & $\mathrm{Mpc}$ & 0.116 \\
{$\left[R_{\mathrm{H}}\right]_{\mathrm{EM}}$} & $\mathrm{Mpc}$ & 0.202 \\
{$\left[R_{\mathrm{H}}\right]_{\mathrm{K}}$} & $\mathrm{Mpc}$ & 0.210 \\
{$[\alpha]_{\mathrm{MG}}$} & - & 0.870 \\
{$\left[t_{\mathrm{cross}} H_{0}\right]$} & - & 0.208 \\
\hline
\end{tabular}

its mass density is about $1.8 \times 10^{11} M_{\odot} \mathrm{Mpc}^{-3}$. It can be seen that this value is in the order of that calculated for the sphere of $5 \mathrm{Kpc}$ in the previous paragraph. This result leads us to think that in the $5 \mathrm{Mpc}$ sphere, there could be low-mass dark halo regions without baryonic matter (see: Tully et al. 2002; Tully 2005), with similar mass density to that of the region of galaxies and systems of galaxies. A dark cosmic "ocean", where dark matter is distributed homogeneously, would not be consistent with the above results; it would imply an excess of mass in the layer.

\subsection{Masses of galaxy complexes from sample masses of galaxy groups}

In this subsection, we give mass estimations of galaxy complexes by summing sample masses of the groups which constitute them. Rows 4 and 5 of Table 11 present maximum and minimum values of those sums. Proposed sample masses for nearly all the groups composing the complexes under study are shown in Tables 9 and 10. Results for NGC 2403 are not shown in the tables because this system is considered to be a non-virialized group.

With respect to the Local Group (i.e. the Milky Way - M 31 galaxy complex), the mass appearing in rows 4 and 5 of Table 11 comes from assuming radial orbits for the Milky Way group and isotropy for M 31 in models $\left[R_{\mathrm{H}}\right]_{\mathrm{EM}},[\alpha]_{\mathrm{MG}}$. This leads to a mass ratio 4.1:5 for the Milky Way and M31 groups. As can be seen, the result is close to $M^{\mathrm{VIR}}$ shown in Table 11 for the Local Group.

With respect to M 81 - NGC 2403, we preferred to study the whole complex in this subsection. The reason is that NGC 2403 is not virialized, which means that sample masses derived from steady equations are questionable. In Table 12, we show some characteristics of this complex. The study was made for the 29 galaxies considered by Karachentsev (2005) as belonging to the complex; these represent 25 galaxies from the M 81 group together with 4 from the NGC 2403 group (all the galaxies are addressed, except NGC 2366). According to the value of $\left[t_{\text {cross }} H_{0}\right]$, shown in the last row of Table 12, we can say that this complex is virialized.

In Table 13, power-like mass distribution parameters (see Sect. 4.1) are shown for the M 81 - NGC 2403 complex. They have been calculated for the four harmonic radius models discussed in this paper. From this table, it can be seen that the model leading to an $R$, which best fits $R_{\mathrm{s}}$ (see table note), is the model $\left[R_{\mathrm{H}}\right]_{\mathrm{EM}}$. 
Table 13. Power-like mass distribution parameters for the M81 NGC 2403 complex.

\begin{tabular}{lcc}
\hline \hline Model & $p$ & $R[\mathrm{Mpc}]$ \\
\hline$\left[R_{\mathrm{H}}\right]_{\mathrm{PM}}$ & 1.270 & 0.476 \\
{$\left[R_{\mathrm{H}}\right]_{\mathrm{MG}}$} & 1.235 & 0.481 \\
{$\left[R_{\mathrm{H}}\right]_{\mathrm{EM}}$} & 0.581 & 0.724 \\
{$\left[R_{\mathrm{H}}\right]_{\mathrm{K}}$} & 0.585 & 0.721 \\
\hline
\end{tabular}

Notes. $R_{\mathrm{S}}=0.883 \mathrm{Mpc}$.

Table 14. Virial mass and projected and sample masses, assuming isotropy for the M 81 - NGC 2403 complex.

\begin{tabular}{cccc}
\hline \hline Model & $\left\{M_{\mathrm{vir}}\right\}$ & $\left\{M_{\mathrm{proj}}\right\}_{\beta=0}$ & $\left\{M_{\mathrm{s}}\right\}_{\beta=0}$ \\
\hline$\left[R_{\mathrm{H}}\right]_{\mathrm{EM}},[\alpha]_{\mathrm{MG}}$ & 11.81 & 22.70 & 9.10 \\
{$\left[R_{\mathrm{H}}\right]_{\mathrm{EM}},[\alpha]_{\mathrm{EM}}$} & 11.81 & 39.52 & 8.23 \\
\hline
\end{tabular}

Notes. Masses are in units of $10^{11} M_{\odot}$.

In Table $14,\left\{M_{\text {vir }}\right\},\left\{M_{\text {proj }}\right\}_{\beta=0}$ and $\left\{M_{\mathrm{s}}\right\}_{\beta=0}$ obtained from models $\left[R_{\mathrm{H}}\right]_{\mathrm{EM}},[\alpha]_{\mathrm{MG}}$, and $\left[R_{\mathrm{H}}\right]_{\mathrm{EM}},[\alpha]_{\mathrm{EM}}$ are shown. $\left\{M_{\mathrm{s}}\right\}_{\beta=0}$ results are also shown in rows 4 and 5 of Table 11 as the proposed maximum and minimum values. Both sample mass estimations are somewhat smaller than the value of $M^{\mathrm{VIR}}$ given in Table 11 for the M 81-NGC 2403 complex. In any case, it should be taken into account that the results shown in Table 11 from turn-around radius measurements are for an average group. The sample mass estimations presented in Table 14 can be compared with those proposed in Tables 9 and 10 for the M 81 group. Thus, from the model $\left[R_{\mathrm{H}}\right]_{\mathrm{EM}},[\alpha]_{\mathrm{MG}}$, the mass contribution of NGC 2403 would be about $0.4 \times 10^{11} M_{\odot}$; i.e. the indicative mass of the group. In the case of considering the model $\left[R_{\mathrm{H}}\right]_{\mathrm{EM}},[\alpha]_{\mathrm{EM}}$, the NGC 2403 mass contribution would be about $1.2 \times 10^{11} M_{\odot}$.

In Table 11, we show lower and upper mass estimations for the Cen A - M 83 complex, obtained from sample masses proposed for the Centaurus A and M 83 groups (see Tables 9 and 10). All estimations assume isotropy for the groups. The lower mass estimation comes from using the $\left[R_{\mathrm{H}}\right]_{\mathrm{MG}},[\alpha]_{\mathrm{EM}}$ model for Cen $\mathrm{A}$, and the $\left[R_{\mathrm{H}}\right]_{\mathrm{EM}},[\alpha]_{\mathrm{EM}}$ model for $\mathrm{M} 83$; for the upper mass estimations, we used results from the $\left[R_{\mathrm{H}}\right]_{\mathrm{MG}},[\alpha]_{\mathrm{MG}}$ and $\left[R_{\mathrm{H}}\right]_{\mathrm{EM}},[\alpha]_{\mathrm{MG}}$ models for Cen $\mathrm{A}$ and M 83, respectively. The mass estimations shown in Table 11 for this complex are in agreement with $M^{\mathrm{VIR}}$.

The IC 342 and Maffei groups do not permit isotropy. The sample masses shown in Tables 9 and 10 for these groups are those that correspond to the whole range of permitted values. In Table 11, we show the minimum and maximum sums of these sample masses. These results are in agreement with $M^{\mathrm{VIR}}$, but are smaller than its central value.

The minimum and maximum mass estimations averaged in the number of complexes are shown in rows 4 and 5 of Col. 7 of Table 11. As can be seen, they are in agreement with that obtained for $M^{\mathrm{VIR}}$. These averaged masses are those that correspond to the average complex.

\section{Conclusions}

The aim of this paper is to include surface effects and cosmological constant terms in mass estimators of galaxy systems. For the sake of simplicity, spherical symmetry, steady and static conditions, and a constant anisotropy parameter are assumed; galaxies are taken as tracers of mass.
From equations in the continuous model obtained by integrating the product of the radial Jeans equation with $r$ and $r^{2}$ over a volume, we have proposed two equations to deal with a sample of $N$ galaxies. The gravitational term of the first equation (known as the virial theorem) comes from the trace of the Chandrasekhar's gravitational potential energy tensor; hence, it depends on the mass enclosed up to the position of each galaxy. The gravitational term of the second equation also depends on mass distribution. The dependence on the anisotropy parameter, $\beta$, appears in the kinetic term of the second equation; this term shows an increasing behaviour with $\beta$.

Surface terms appear when a system extends beyond the volume of the galaxy sample. When these terms are neglected, masses derived from the two equations are commonly called virial mass, $\left\{M_{\mathrm{vir}}\right\}$, and projected mass, $\left\{M_{\text {proj }}\right\}$, respectively. The surface terms of both equations are related by the distance of the farthest galaxy from the mass centre of the sample. This allows an equation to be obtained, which estimates the mass contained in the volume of the sample, $\left\{M_{\mathrm{s}}\right\}$, without having to calculate these terms. This mass, which we call the sample mass, must be smaller than, or equal to, both the virial and projected masses.

The nearest galaxy groups located inside $5 \mathrm{Mpc}$ (the Milky Way, M 31, M 81, NGC 2403, Cantaurus A, M 83, IC 342, Maffei, Sculptor Filament, and Canes Venatici I Cloud), are addressed in this paper. These groups contain a massive galaxy whose mass represents the majority of the galaxy mass. This fact allows the equations to be simplified.

Various models have been explored for mass distributions. These models assume central point mass (PM), the same mass for companions to the main galaxy (MG), and equal mass for all galaxies (EM). For each galaxy group, just one model is chosen to handle $\left\{M_{\text {vir }}\right\}$; and, models MG and EM are explored in $\left\{M_{\text {proj }}\right\}$. Power-like mass continuous distributions, which depend on two parameters, are used in the selection process (see Sect. 4.1). Parameters are not found for NGC 2403, Sculptor, and Canes Ventatici I; this may reflect the fact that these groups are not virialized.

Our model selection process seems to indicate that the gravitational term of the virial theorem is affected more by the galaxy positions than by the different mass contributions of the galaxies. We have seen that some groups with a massive main galaxy are better represented by models EM (the Milky Way, M 31, M 81, and M 83 groups). However, other groups with a main galaxy but with an appreciable mass contribution from companions, are better represented by models MG (Centaurus A and IC 342).

For the models addressed in this work, the Milky Way, M 31, and IC 342 show a virial mass smaller that any projected mass. M 81, M 83, and Centaurus A also present $\left\{M_{\text {vir }}\right\}$ smaller than $\left\{M_{\text {proj }}\right\}$, but only when models EM are used for projected masses. We note that Maffei shows rather more $\left\{M_{\text {vir }}\right\}$ than any $\left\{M_{\text {proj }}\right\}$ estimated, taking models MG; when projected masses are derived from models EM, only those fulfilling $\beta>0.95$ are greater than the virial mass of this group. Differences between $\left\{M_{\mathrm{vir}}\right\}$ and $\left\{M_{\text {proj }}\right\}$ indicate that surface terms make an appreciable contribution.

With respect to contributions of the cosmological constant terms to virial mass estimations, $\left[f_{\text {vir }}\right]$, we have seen that the Milky Way, M31, M 81, M 83, Maffei, and Centaurus A show $\left[f_{\text {vir }}\right]<0.03$; but, that IC 342, Sculptor and CVn I present $0.08 \leq\left[f_{\text {vir }}\right] \leq 0.11$. NGC 2403 shows the greatest value, $\left[f_{\text {vir }}\right] \approx 0.47$. These results suggest that non-virialized groups present greater values of $\left[f_{\text {vir }}\right]$ than virialized galaxy groups; i.e. there is a greater quotient between the mean square projected separation of galaxies from the principal galaxy, $\left[R_{\perp}^{2}\right]$, 
and the square dispersion of the line of sight velocities in the group, $\left[\sigma_{\text {los }}^{2}\right]$. Thus, the studied virialized groups present $\left[R_{\perp}^{2}\right] /\left[\sigma_{\text {los }}^{2}\right]<1.5 \times 10^{-5} \mathrm{Mpc}^{2} \mathrm{~km}^{-2} \mathrm{~s}^{2}$, while the non-virialized groups show $\left[R_{\perp}^{2}\right] /\left[\sigma_{\text {los }}^{2}\right]>4 \times 10^{-5} \mathrm{Mpc}^{2} \mathrm{~km}^{-2} \mathrm{~s}^{2}$, being $2.6 \times$ $10^{-4} \mathrm{Mpc}^{2} \mathrm{~km}^{-2} \mathrm{~s}^{2}$ for NGC 2403 .

The contributions of cosmological constant terms to projected mass, $\left[f_{\text {proj }}\right]_{\beta}$, are similar or even smaller than $\left[f_{\text {vir }}\right]$. They show a decreasing behavior with $\beta$.

With respect to our sample mass estimator, we have shown that $\left\{M_{\mathrm{s}}\right\}$ can be expressed as a function of $\left\{M_{\mathrm{vir}}\right\},\left\{M_{\mathrm{proj}}\right\}$ and a parameter denoted by $[\gamma]$. This parameter is related to the quotient between the harmonic radius of a group and the distance of the farthest galaxy from the main galaxy in the group. We have seen that $[\gamma]<1$ for virialized groups; but for non-virialized groups, $[\gamma]$ can take values greater than unity. From our study, we have realized that $\left\{M_{\mathrm{s}}\right\}$ is restricted to a range of anisotropy parameters. This range is fixed by the fact that surface terms must be positive or zero, and by the fact that the sample mass must be greater than or equal to the galaxy mass of the sample.

We note that Maffei has allowed only Keplerian averaged orbits close to radial ones. We have also seen that for some models, the M 83 group shows only anisotropy parameters where the radial velocity dispersion dominates. The opposite behavior occurs with IC 342; for this group, tangential velocity dispersion dominates the anisotropy parameters for all the models. The rest of the groups show a wide range of allowed anisotropy parameters.

The virialized groups being studied show positive contributions of the cosmological constant terms to sample masses, $\left[f_{\mathrm{s}}\right]$. They are smaller than 0.02 for the Milky Way, M 31, M 81, and Maffei; while, for M 83, Centaurus A, and IC 342, they range from 0.01 up to 0.13 . With respect to the groups with questionable virialization, some models present negative values of $\left[f_{\mathrm{s}}\right]$; in any case, their modulus is much greater than that shown by the majority of the virialized groups. These results are derived assuming steady conditions; hence, they are questionable for nonvirialized groups.

For each virialized cluster, sample masses have been selected from the whole range of allowed masses. In the absence of information about the anisotropy parameter of the group, isotropy is assumed. If isotropy is not allowed, the whole range is considered.

With respect to the Milky Way group, the existence of the polar Magellanic Stream leads us to think about strongly elongated orbits. Thus, we have chosen $\beta=1$ for the Milky Way. For M 31, we have taken $\beta=0$. From these assumptions, our results for these galaxy groups are $3.8 \times 10^{11} M_{\odot}$ for the Milky Way and $4.6 \times 10^{11} M_{\odot}$ for M 31 . These have been derived assuming model EM for $\left\{M_{\mathrm{vir}}\right\}$, and model MG for $\left\{M_{\text {proj }}\right\}$. These values lead to a mass ratio of 4.1:5, which is in agreement with the expected mass ratio of 4:5.

Isotropy is assumed for M 81 and M 83. Then, using model EM for $\left\{M_{\mathrm{vir}}\right\}$, the sample mass estimations from models MG and EM for $\left\{M_{\text {proj }}\right\}$ are: $8.7 \times 10^{11} M_{\odot}$ and $7.0 \times 10^{11} M_{\odot}$ for M 81 , and $10.0 \times 10^{11} M_{\odot}$ and $6.6 \times 10^{11} M_{\odot}$ for M 83 .

Maffei only allows anisotropy parameters that fulfill $0.95 \leq$ $\beta \leq 1$ from models EM for $\left\{M_{\text {vir }}\right\}$ and $\left\{M_{\text {proj }}\right\}$. For this range of $\beta$, $5.3 \times 10^{11} M_{\odot} \leq\left\{M_{\mathrm{s}}\right\} \leq 5.6 \times 10^{11} M_{\odot}$.

Isotropy is also assumed for Centaurus A. Sample masses from model MG for $\left\{M_{\mathrm{vir}}\right\}$ and models MG and EM for $\left\{M_{\text {proj }}\right\}$ are $18.8 \times 10^{11} M_{\odot}$ and $13.4 \times 10^{11} M_{\odot}$.

According to our results, isotropy is not allowed in IC 342. From model MG for $\left\{M_{\text {vir }}\right\}$ and models MG and EM for $\left\{M_{\text {proj }}\right\}$, sample masses fulfilling $1.1 \times 10^{11} M_{\odot} \leq\left\{M_{\mathrm{s}}\right\} \leq 1.7 \times 10^{11} M_{\odot}$ and $1.1 \times 10^{11} M_{\odot} \leq\left\{M_{\mathrm{s}}\right\} \leq 1.5 \times 10^{11} M_{\odot}$ are estimated for the whole range of allowed $\beta$.

When mass estimations are compared, we see that $\left\{M_{\mathrm{s}}\right\}$ is closer to $\left\{M_{\text {vir }}\right\}$ than to $\left\{M_{\text {proj }}\right\}$. The M $81, \mathrm{M} 83$, and Maffei groups show the closest $\left\{M_{\mathrm{s}}\right\}$ to $\left\{M_{\mathrm{vir}}\right\}$ and $\left\{M_{\text {proj }}\right\}$. For the M 81 and M 83 groups, models MG for projected mass lead to sample masses, which represent more than $90 \%$ of virial masses and more than $80 \%$ of projected masses; with respect to models EM for projected mass, they account for more than $70 \%$ and $35 \%$, respectively. For the Maffei group, $\left\{M_{\mathrm{s}}\right\}$ is more than $95 \%$ of $\left\{M_{\mathrm{vir}}\right\}$ and more than $87 \%$ of $\left\{M_{\text {proj }}\right\}$. The Milky Way and IC 342 present the greatest differences between masses: $\left\{M_{\mathrm{s}}\right\}$ ranges from $38 \%$ to $60 \%$ of $\left\{M_{\mathrm{vir}}\right\}$, and is smaller than $19 \%$ of $\left\{M_{\text {proj }}\right\}$.

In this paper, turn-around masses, $M^{\mathrm{TA}}$, and virialized masses, $M^{\text {VIR }}$, of virialized galaxy complexes located up to $5 \mathrm{Mpc}$ have also been estimated from measurements of turnaround radii, $r^{\mathrm{TA}}$. Turn-around radii have been taken from works by Karachentsev et al. (2002a,b,c, 2003a,b,c,d). To calculate $M^{\mathrm{TA}}$ and $M^{\mathrm{VIR}}$ from $r^{\mathrm{TA}}$, results by Membrado \& Pacheco (2014) have been used. Our results differ from those obtained by Karachentsev (2005) because he used the Lynden-Bell (1981) and Sandage (1986) relation, which does not take into account the cosmological constant effect.

For the Milky Way - M31 complex, we have estimated that $M^{\mathrm{TA}}=(17.4 \pm 5.6) \times 10^{11} M_{\odot}$ and $M^{\mathrm{VIR}}=(8.9 \pm 2.8) \times 10^{11} M_{\odot}$; for $\mathrm{M} 81-\mathrm{NGC} 2403, M^{\mathrm{TA}}=(24.3 \pm 4.9) \times 10^{11} M_{\odot}$ and $M^{\mathrm{VIR}}=(12.5 \pm 2.5) \times 10^{11} M_{\odot}$; for Centaurus A $-\mathrm{M} 83$, $M^{\mathrm{TA}}=(42.0 \pm 15.0) \times 10^{11} M_{\odot}$ and $M^{\mathrm{VIR}}=(21.5 \pm 7.7) \times 10^{11} M_{\odot}$; and, for IC $342-$ Maffei, $M^{\mathrm{TA}}=(15.3 \pm 5.1) \times 10^{11} M_{\odot}$ and $M^{\mathrm{VIR}}=(7.9 \pm 2.6) \times 10^{11} M_{\odot}$.

The above galaxy complexes are contained in a sphere of $5 \mathrm{Mpc}$. In this volume, 233 galaxies have been observed. Using the turn-around mass-to-luminosity ratio for these complexes as an upper limit for the mass-to-luminosity ratio in the sphere, we have seen that mass density in the sphere should be smaller than $76 \%$ of the mass density of matter background in the present Universe. As already pointed out by Karachentsev (2005), it would be necessary to assume the existence of another component of dark matter.

If mass density were proportional to luminosity density at different scales, an estimation of the mean mass-to-luminosity ratio in the Universe would be $[M / L]_{0} \approx 190 M_{\odot} L_{\odot}^{-1}$. When this result is applied to the sphere of $5 \mathrm{Mpc}$, it is calculated that its mass density would be about 4.5 times greater than that of the matter background.

All groups addressed in this paper are located in a layer of $\pm 1 / 3 \mathrm{Mpc}$ around the local "pancake". We have seen that the mass density of the region that contains the turn-around volumes of the four virialized complexes and the two expanding groups and the volume of the layer, which is not occupied by them, is about 4.7 times that of the matter background at present. For the sphere of $5 \mathrm{Mpc}$, this result could imply the existence of regions occupied by low-mass dark haloes with a density similar to that of the region of galaxies and galaxy systems. Dark matter distributed homogeneously would not be allowed because it would imply an excess of mass in the layer.

Virialized masses, $M^{\mathrm{VIR}}$, calculated from measured turnaround radii of galaxy complexes can also be compared with the sum of the sample mass estimations of their groups. In this work, we have summed the greatest sample mass estimations of those proposed for deriving $\max \left(\sum\left\{M_{\mathrm{s}}\right\}\right)$, and the lowest ones for calculating $\min \left(\sum\left\{M_{\mathrm{s}}\right\}\right)$. 
For the Milky Way - M31 complex, since just one value is proposed for the sample mass of each group, $8.4 \times 10^{11} M_{\odot}$ is obtained for the mass of the complex.

As NGC 2403 is a non-virialized galaxy group, its sample mass estimations from our models are questionable. Thus, we preferred to estimate the sample mass of the whole complex M 81 - NGC 2403. Thus, we obtained $9.1 \times 10^{11} M_{\odot}$ and $8.2 \times 10^{11} M_{\odot}$ from two models.

With respect to Centaurus A - M 83, we have obtained $\max \left(\sum\left\{M_{\mathrm{s}}\right\}\right)=28.8 \times 10^{11} M_{\odot}$ and $\min \left(\sum\left\{M_{\mathrm{s}}\right\}\right)=20.0 \times$ $10^{11} M_{\odot}$.

Finally, when the whole range of sample mass estimations is taken into account for the groups making up the complex IC 324 - Maffei, the maximum and minimum values for the sum of sample masses are $7.3 \times 10^{11} M_{\odot}$ and $6.4 \times 10^{11} M_{\odot}$, respectively.

As can be seen, for all the complexes studied, except M 81 NGC 2403, the above results are in agreement with virialized masses derived from turn-around radii. The values for M 81 NGC 2403 are smaller than $M^{\mathrm{VIR}}$; in any case, we must bear in mind that virialized masses are calculated for the average cluster.

A final comparison can be made among $M^{\mathrm{VIR}}, \max \left(\sum\left\{M_{\mathrm{s}}\right\}\right)$ and $\min \left(\sum\left\{M_{\mathrm{s}}\right\}\right)$ averaged in the number of complexes. These averages are $(12.7 \pm 3.9) \times 10^{11} M_{\odot}, 13.4 \times 10^{11} M_{\odot}$, and $10.8 \times$ $10^{11} M_{\odot}$, respectively. As can be seen the two latter average quantities are in agreement with the former.

\section{References}

Bahcall, J. N., \& Tremaine, S. 1981, ApJ, 244, 805

Binney, J., \& Tremaine, S. 1987, Galactic dynamics (Princeton Univ. Press)

Blanton, M. R., Hogg, D. W., Bahcall, N. A., et al. 2003, ApJ, 592, 819

Carlberg, R. G., Yee, H. K. C., Ellingson, E., et al. 1996, ApJ, 462, 32

Chandrasekhar, S. 1942, Principles of stellar dynamics (Dover Books on Physics)

Chernin, A. D., Teerikorpi, P., Valtonen, M. J., et al. 2012, Grav. Cosmol., 18, 1 Crone, M. M., Evrard, A. E., \& Richstone, D. O. 1994, ApJ, 434, 402

Geller, M. J. 1984, in Clusters and Groups of Galaxies, eds. F. Mardirossian, G. Giuricin, \& M. Mezzetti, Astrophys. Space Sci. Libr., 111, 353
Gott, III, J. R., \& Turner, E. L. 1977, ApJ, 213, 309

Gunn, J. E., \& Gott, III, J. R. 1972, ApJ, 176, 1

Heisler, J., Tremaine, S., \& Bahcall, J. N. 1985, ApJ, 298, 8

Huchra, J. P., \& Geller, M. J. 1982, ApJ, 257, 423

Karachentsev, I. D. 2005, AJ, 129, 178

Karachentsev, I. D., \& Nasonova, O. G. 2010, MNRAS, 405, 1075

Karachentsev, I. D., Dolphin, A. E., Geisler, D., et al. 2002a, A\&A, 383, 125

Karachentsev, I. D., Sharina, M. E., Dolphin, A. E., et al. 2002b, A\&A, 385, 21

Karachentsev, I. D., Sharina, M. E., Makarov, D. I., et al. 2002c, A\&A, 389, 812

Karachentsev, I. D., Grebel, E. K., Sharina, M. E., et al. 2003a, A\&A, 404, 93

Karachentsev, I. D., Makarov, D. I., Sharina, M. E., et al. 2003b, A\&A, 398, 479

Karachentsev, I. D., Sharina, M. E., Dolphin, A. E., \& Grebel, E. K. 2003c, A\&A, 408, 111

Karachentsev, I. D., Sharina, M. E., Dolphin, A. E., et al. 2003d, A\&A, 398, 467

Karachentsev, I. D., Karachentseva, V. E., Huchtmeier, W. K., \& Makarov, D. I. 2004, AJ, 127, 2031

Limber, D. N., \& Mathews, W. G. 1960, ApJ, 132, 286

Liske, J., Lemon, D. J., Driver, S. P., Cross, N. J. G., \& Couch, W. J. 2003, MNRAS, 344, 307

Lynden-Bell, D. 1981, The Observatory, 101, 111

Membrado, M., \& Aguerri, J. A. L. 2004, A\&A, 424, 415

Membrado, M., \& Pacheco, A. F. 2014, A\&A, 567, A37

Page, T. 1952, ApJ, 116, 63

Peebles, P. J. E. 1980, The large-scale structure of the Universe (Princeton Univ. Press)

Rines, K., \& Diaferio, A. 2006, AJ, 132, 1275

Rines, K., Geller, M. J., Kurtz, M. J., \& Diaferio, A. 2003, AJ, 126, 2152

Rood, H. J. 1981, Rep. Prog. Phys., 44, 1077

Rood, H. J., \& Dickel, J. R. 1978, ApJ, 224, 724

Rood, H. J., Rothman, V. C. A., \& Turnrose, B. E. 1970, ApJ, 162, 411

Sandage, A. 1986, ApJ, 307, 1

Schwarzschild, M. 1954, AJ, 59, 273

Spergel, D. N., Verde, L., Peiris, H. V., et al. 2003, ApJS, 148, 175

The, L. S., \& White, S. D. M. 1986, AJ, 92, 1248

Tully, R. B. 2005, ApJ, 618, 214

Tully, R. B., Somerville, R. S., Trentham, N., \& Verheijen, M. A. W. 2002, ApJ, 569,573

Wolf, R. A., \& Bahcall, J. N. 1972, ApJ, 176, 559

Zwicky, F. 1933, Helvetica Physica Acta, 6, 110 